\title{
Point Defects, Topological Chirality, and Singularity Theory in Cholesteric Liquid-Crystal Droplets
}

\author{
Joseph Pollard, ${ }^{1}$ Gregor Posnjak, ${ }^{2}$ Simon Čopar, ${ }^{3}$ Igor Muševič, ${ }^{2,3}$ and Gareth P. Alexander, ${ }^{4, *}$ \\ ${ }^{1}$ Mathematics Institute, Zeeman Building, University of Warwick, Coventry, CV4 7AL, United Kingdom \\ ${ }^{2}$ Condensed Matter Department, Jožef Stefan Institute, Jamova 39, 1000 Ljubljana, Slovenia \\ ${ }^{3}$ Department of Physics, Faculty of Mathematics and Physics, University of Ljubljana, \\ Jadranska 19, 1000 Ljubljana, Slovenia \\ ${ }^{4}$ Department of Physics and Centre for Complexity Science, University of Warwick, \\ Coventry, CV4 7AL, United Kingdom
}

(Received 12 November 2018; revised manuscript received 6 February 2019; published 8 April 2019)

\begin{abstract}
We provide a characterization of point defects in droplets of cholesteric liquid crystal, using a combination of experiment, simulation, and theoretical analysis. These droplets display a range of structures including realizations of defects with high topological charge and arrangements of multiple defects in "topological molecules." We show that there are certain defects that are incompatible with a uniform sense of chiral twisting for topological reasons. Furthermore, those defects that are compatible with twist of a single handedness are shown to have the structure of the gradient field of an isolated critical point and, hence, are described by singularity theory. We show that the mathematical tools of singularity theory reproduce, with excellent agreement, the experimental observations of high charge defects and topological molecules. Our results have implications beyond liquid crystal droplets in characterizing chiral materials and their topology in general.
\end{abstract}

DOI: 10.1103/PhysRevX.9.021004

Subject Areas: Soft Matter

\section{INTRODUCTION}

The character of materials is conveyed by their defects: They control strength or fragility [1], determine elastic interactions [2,3], mediate self-assembly [4-6], and precipitate phase transitions [7-11]. The widespread influence of defects derives from their high energetic cost, strong elastic distortions, and topological nature. This is especially true in the liquid crystalline mesophases, whose defects have often offered key insights and motivated the naming of textures. Moreover, liquid crystals offer a versatile setting for studying topological phenomena, often with relevance across multiple disciplines, including cosmological strings [12], biological tissues and morphogenesis $[13,14]$, and magnetic skyrmion textures [15-17]. Chiral materials furnish an especially rich source of topological and geometric phenomena, and chirality is also at the heart of one of the fundamental contrasts given in liquid crystals: between smectics and cholesterics. In de Gennes's famous analogy [18], their contrast is equivalent to the difference

\footnotetext{
*Corresponding author. g.p.alexander@warwick.ac.uk.

Published by the American Physical Society under the terms of the Creative Commons Attribution 4.0 International license. Further distribution of this work must maintain attribution to the author(s) and the published article's title, journal citation, and DOI.
}

between metals and superconductors, with the expulsion of twist from smectics being analogous to the Meissner effect. Here, we emphasize a new paradigm where the defects in chiral materials are forced to have the local structure of a smectic in order to be chiral.

The character of point defects in cholesteric liquid crystals (or magnetic skyrmion textures) appears not to have been considered previously, apart from recent experimental work realizing them [19-21]. This contrasts with the situation in nematics where point defects known colloquially as hedgehogs have been extensively studied over several decades [2-4,22-27]. They can be generated deliberately as satellite defects to colloidal inclusions, or in droplets, with normal anchoring. For spherical colloids, point defects form elastic dipoles and facilitate self-assembly of colloidal chains and lattices [5], while in droplets, transitions between defect states produced by changes in boundary conditions [24] can provide highly sensitive sensors [28,29]. Point defects in nematics are classified by an integer known as the topological charge, or degree [30]. Normal anchoring boundary conditions on a surface of genus $g$ (the number of "holes" or "handles") correspond to a topological charge $1-g$ and induce compensating point defects of the same total topological charge in liquid crystal surrounding colloidal inclusions [31] or inside toroidal droplets [32]. In addition to their own phenomenology, the topological character of point defects has also provided fundamental insight into 
disclination loops and their classification [30,33,34]. The lack of a similar body of work for point defects in cholesterics represents a gap in our understanding of chiral materials; closing it will provide considerable insight into cholesterics and also skyrmion textures of chiral ferromagnets.

In recent experiments [19-21], point defects were created in spherical droplets of cholesteric liquid crystal with normal surface anchoring and shown to have markedly different properties from their nematic counterparts. These properties included point defects of topological charge +1 , $-1,-2$, and -3 , the higher charge defects observed for the first time, as well as arrangements of multiple defects into "topological molecules." Defects were located both in the center of the droplet and in close proximity to its surface. The latter are not the surface defects associated with planar anchoring [24] but a feature of the cholesteric order. Here, we provide a theoretical description of point defects in cholesterics that reproduces the structures observed experimentally and explain the topological character of chiral point defects. The main feature of this description is that the structure is determined by a gradient field: Gradient fields are more commonly associated with smectics, whose basic properties include the fundamental absence, or expulsion, of chirality $[10,18]$, so that the emergence of a gradient structure appears surprising. It establishes a previously unnoticed nexus between cholesterics and smectics.

Generically, point defects are not chiral and sit on surfaces separating domains of opposite handedness; there is then an energetic drive to expel those defects from the interior. As far as we are aware, this simple statement appears not to have been said explicitly previously. Nonetheless, there are defects that are compatible with a fully chiral surrounding; for those, their local structure is that of a gradient field with isolated critical point and the chirality provided by higherorder terms in a Taylor series. The type of critical point provides a label for the defect that refines the topological charge. Applying a fundamental mathematical result in topology, we show that radial-like vector fields necessarily contain regions with both handedness. In chiral materials, the region with the wrong handedness becomes localized for energetic reasons and is a novel topological soliton.

Defects with higher topological charge are associated with the gradient fields of degenerate critical points, giving a new physical application for singularity theory [35-37]. The formalism of singularity theory allows us to identify the defects of high topological charge $(-2$ and -3$)$ seen experimentally and to study the ways in which complex high-charge defects can break apart into simpler pieces, yielding excellent agreement with the topological molecules we observe. We further identify the "chemical bonds" of these topological molecules with natural structural defects of the cholesteric twist and geometric degeneracies in the gradient field of the singularity.

A close similarity exists between the textures of cholesteric liquid crystals and those of chiral ferromagnets
$[17,38,39]$. This similarity arises because both systems are driven by a coupling that promotes twist in the nematic or magnetic alignment; this coupling is the addition of a chiral dopant in the liquid crystal case and the Dzyaloshinskii-Moriya interaction in the ferromagnetic one. As a result, the description we give here for point defects in cholesterics applies also to the monopoles that have been seen in skyrmionic magnets [40].

The remainder of this paper is organized as follows: In Sec. II, we give a brief summary of the topological characterization of point defects, including those that come from the gradient of a function. In Sec. III, we provide summary details of experiments and simulations of cholesteric droplets that are described throughout the remainder of the text. The structure and properties of generic point defects in cholesterics are then presented in Secs. IV and V. In Sec. VI, we discuss the behavior of the twist in a boundary layer at the droplet surface. In Sec. VII, we extend the generic description of chiral defects to those with higher topological charge using singularity theory; this section also begins with a summary of some concepts in singularity theory. In Sec. VIII, we describe a connection between chiral defects and curl eigenfields that provides an alternative perspective. In Sec. IX, we describe degeneracies in the twisting direction and how they relate to the structure of the singularity in chiral point defects. The paper ends in Sec. X with a discussion.

\section{CHARACTERIZATION OF POINT DEFECTS AND TOPOLOGICAL CHARGES}

Liquid crystals are anisotropic fluids described by an average molecular orientation called the director field, $\mathbf{n}$. This is a unit magnitude vector; however, the symmetry of the nematic phase is apolar rather than vectorial, meaning that $\mathbf{n}$ and $-\mathbf{n}$ are physically equivalent. Point defects known colloquially as hedgehogs are isolated points where the molecular orientation is undefined and discontinuous and that are classified by an integer topological charge. In nematic liquid crystals, the definition of the topological charge is complicated by their apolar symmetry. The topological charge of a point defect reflects the number of times the director sweeps out all orientations of the unit sphere on any surface surrounding the defect. For example, the radial "hedgehog" $\mathbf{n}=\mathbf{x} /|\mathbf{x}|$ takes on every orientation exactly once on a spherical surface centered on the origin and has topological charge +1 . In general, for any surface $S$ with local coordinates $(u, v)$, the topological charge $Q$ is given by the integral

$$
Q=\frac{1}{4 \pi} \int_{S} \mathbf{n} \cdot\left(\frac{\partial \mathbf{n}}{\partial u} \times \frac{\partial \mathbf{n}}{\partial v}\right) d u d v .
$$

We remark that the value of this integral changes sign under the replacement $\mathbf{n} \rightarrow-\mathbf{n}$ corresponding to the apolar symmetry of nematics. This is the infamous sign ambiguity of 
topological charge in liquid crystals [30] that is responsible for many of their more subtle, and more interesting, properties.

In the context of our current investigation, the sign ambiguity in the topological charge is entirely resolved by the choice we make for the boundary conditions at the droplet surface, together with a restriction to consider only point defects in the director, i.e., to exclude any disclination lines. In this setting, the director is orientable, even though it is not explicitly oriented, and unambiguous signs for topological charges follow from adopting a particular choice of orientation and sticking to it consistently. The choice that we make is that the director coincides everywhere with the outward normal direction at the surface of the droplet. This choice also constrains the total topological charge of all point defects in the liquid crystal through the Poincaré-Hopf index theorem: For spherical droplets that we consider here, the total charge is +1 .

We adopt a useful visualization method for presenting complex director fields and identifying topology that comes from the Pontryagin-Thom construction as applied to liquid crystals [41-43]. One chooses a fixed direction $\mathbf{p}$, say, the $z$ direction, and then the texture is depicted by showing the surface in space where the director field is perpendicular to $\mathbf{p}$. If $\mathbf{p}$ is the $z$ direction, this surface will be the set of points where the director is "horizontal." The surface is colored according to the horizontal orientation of the director on it with a full color winding corresponding to a rotation of the director by $\pi$ rather than $2 \pi$, in accordance with the apolar nematic symmetry. Points about which the color winds are defects, whose topological charge is given by the number of full $2 \pi$ windings. The construction is illustrated for the radial hedgehog $\mathbf{n}=\mathbf{x} /|\mathbf{x}|$ in Fig. 1; see Refs. [41-43] for full details.

\section{A. Gradient fields and Morse index}

A special class of vector fields that play a central role in our work is provided by those that are the gradient vector
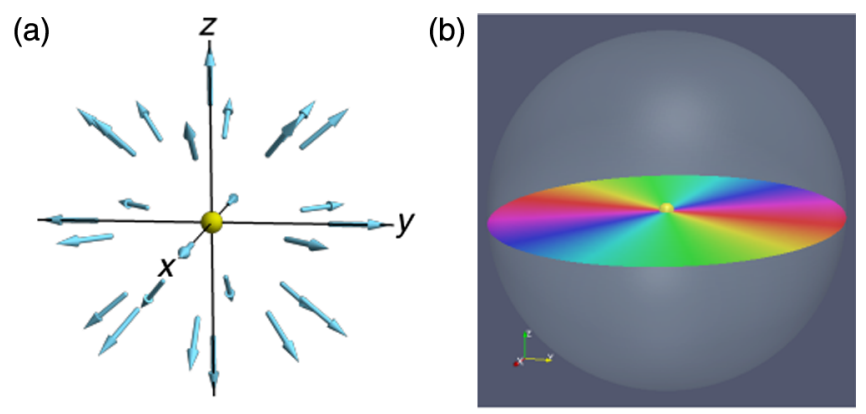

FIG. 1. (a) Schematic director field of a radial point defect (yellow sphere). (b) Visualization using the Pontryagin-Thom construction. The surface is the set of points where $n_{z}=0$ colored by the horizontal orientation. There are two full color windings around the central point defect (yellow sphere) because of the apolar symmetry of nematics. field of a function, i.e., $\mathbf{n} \propto \nabla \phi$. The defects in these gradient vector fields come from the critical points of the function $\phi$, which enjoy a more refined classification than is given by the topological charge alone. Critical points of functions are studied in the branch of mathematics known as Morse theory [44], where it is shown that any generic critical point is equivalent to one of the following normal forms

$$
\begin{aligned}
& \text { index } 0: x^{2}+y^{2}+z^{2}, \\
& \text { index 1: } x^{2}+y^{2}-z^{2}, \\
& \text { index 2: } x^{2}-y^{2}-z^{2}, \\
& \text { index 3: }-x^{2}-y^{2}-z^{2}
\end{aligned}
$$

that are labeled by the number of minus signs called the Morse index. These generic Morse critical points are illustrated in Fig. 2, where we show the local structure of the function and also of its gradient field.

Critical points with Morse index 0 or 2 have topological charge +1 , while those with Morse index 1 or 3 have topological charge -1 . The topological charge can be calculated from the gradient field using Eq. (1) with $\mathbf{n}=\nabla \phi /|\nabla \phi|$. Another method, which is often convenient, comes from the structure of the local level sets of $\phi$ near the critical point. Each level set of $\phi$ is a surface that is singular at the critical value $(\phi=0)$ but smooth for regular values. If we denote by $\chi_{ \pm}$the Euler characteristic of this local set for values just above $(+)$ or below (-) the critical value, then the topological charge is given by [45]

$$
Q=1-\chi_{-}=\chi_{+}-1 .
$$

As an example, for the Morse index 0 critical point shown in Fig. 2, the level sets with $\phi>0$ are spheres $\left(\chi_{+}=2\right)$, while the level sets with $\phi<0$ are empty $\left(\chi_{-}=0\right)$, and it follows that the topological charge is +1 . Likewise, for the Morse index 1 critical point, the level sets with $\phi>0$ are single-sheeted hyperboloids $\left(\chi_{+}=0\right)$, while those with $\phi<0$ are double-sheeted hyperboloids $\left(\chi_{-}=2\right)$, so the topological charge is -1 .

This calculation of the topological charge can also be viewed directly in terms of the director field. Namely, the director field on any spherical surface can be decomposed into a collection of patches where it points "inwards," those where it points "outwards," and a boundary between them where it is tangential. A direct analysis of the gradient fields we consider here gives the topological charge as the number of outward pointing patches minus the number of inward pointing ones; a more complete discussion is given in Ref. [46]. 

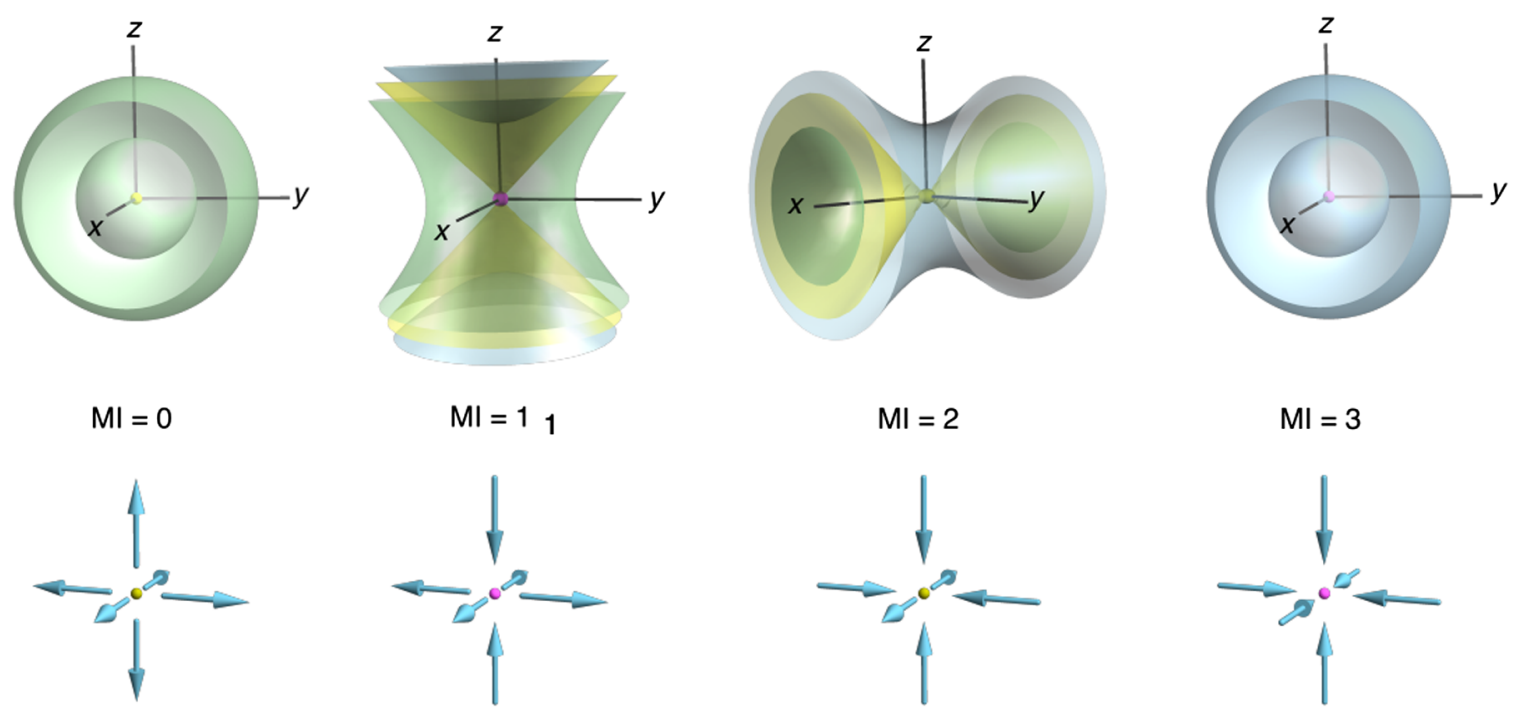

FIG. 2. Illustration of the four types of Morse critical points. Top: Structure of the level sets near the critical point; green for values above the critical value, yellow for the critical value, and blue for values below the critical value. In the case of the Morse index (MI) 0 and 3 critical points, the larger spherical level set is "opened" to provide better visualization of the interior structure. Bottom: Structure of the corresponding gradient fields.

\section{CHOLESTERIC DROPLETS}

In a cholesteric liquid crystal, the director field extremizes the Frank free energy [47]

$$
\begin{aligned}
F= & \int \frac{K_{1}}{2}(\nabla \cdot \mathbf{n})^{2}+\frac{K_{2}}{2}\left(\mathbf{n} \cdot \nabla \times \mathbf{n}+q_{0}\right)^{2} \\
& +\frac{K_{3}}{2}[(\mathbf{n} \cdot \nabla) \mathbf{n}]^{2} d V,
\end{aligned}
$$

where the $K_{i}$ are elastic constants-splay, twist, and bend, respectively - and $q_{0}$ is a chiral coupling constant called the chirality. We shall assume that the cholesteric is right handed, corresponding to $q_{0}>0$. The free energy is minimized by a director with zero splay $(\nabla \cdot \mathbf{n}=0)$ and bend $((\mathbf{n} \cdot \nabla) \mathbf{n}=0)$ but constant nonzero twist $(\mathbf{n} \cdot \nabla \times$ $\left.\mathbf{n}=-q_{0}\right)$ at the preferred rate. In a bulk material without the influence of confinement, the minimum energy configuration is the helical cholesteric ground state $\mathbf{n}=\cos q_{0} z \mathbf{e}_{x}+\sin q_{0} z \mathbf{e}_{y}$ and is periodic with periodicity $p=\pi / q_{0}$ known as the cholesteric half-pitch. Moving along the pitch axis ( $z$ axis), the director rotates at a uniform rate and in the sense of a right-handed screw. Our focus is on spherical droplets of cholesteric liquid crystal with strong normal anchoring conditions at the surface. As we describe in Sec. II, these boundary conditions enforces the existence of topological defects, and both they and the surface anchoring frustrate the preferred helical twisting. Despite this frustration, the energy still favors a right-handed twist $(\mathbf{n} \cdot \nabla \times \mathbf{n}<0)$, and the key question we address is how this energetic preference and frustration resolve themselves.

A natural dimensionless measure of the strength of the chirality in the droplet is the ratio of the droplet diameter $2 R$ to the cholesteric half-pitch, $N=2 R / p$. In the experiments of Refs. [20,21], the typical values were $2 \lesssim N \lesssim 6$; in the simulations shown here, we use values between 2 and 4 . Numerical minimization of Eq. (4) is done using a finitedifference relaxation algorithm on a cubic grid; for simplicity, we adopt a one elastic constant approximation $\left(K_{1}=K_{2}=K_{3}\right)$, and the only relevant parameter is then the dimensionless ratio $N$. Typical simulations are run on a spherical droplet inside a $100^{3}$ grid with Dirichlet boundary conditions and initial conditions provided by the local forms described in Secs. V, VII, and VIII. We find that it is sufficient to specifically initialize only the interior point defect(s), allowing those on the "surface" to emerge naturally through matching to the radial boundary conditions. The use of a vector field for the simulation of the liquid crystal ensures that only point defects arise; in simulations using a $Q$ tensor, such points typically expand into small disclination loops [48]. This approach captures all the phenomenology and major experimental observations; more quantitative comparison can come from including defect core structure, elastic anisotropy, and surface anchoring energy in place of Dirichlet boundary conditions.

Experimental samples are prepared by mixing small amounts of a chiral nematic liquid crystal mixture (1:1 weight ratio of $\mathrm{CCN}-47$ and $\mathrm{CCN}-55$ with $1.7 \% \mathrm{~S}-811$ chiral dopant) into glycerol with $4 \% L-\alpha$-phosphatidylcholine (egg yolk lecithin), which ensures homeotropic anchoring on the droplets of liquid crystal (LC). The samples are heated to isotropic phase above $65^{\circ} \mathrm{C}$ and quickly cooled back to chiral nematic phase. This procedure enables the formation of a diverse range of stable and metastable director structures [20,21], some of which include the degree -2 and -3 defects. The director 
structures are examined by fluorescent confocal polarizing microscopy $[21,49]$, where a small amount of a fluorescent dye $N, N^{\prime}$-bis(2,5-di-tert-butylphenyl)-3,4,9,10-perylenedicarboximide, which aligns with the director, is added to the LC mixture. The dye is excited with linearly polarized light (wavelength $488 \mathrm{~nm}$, linear polarizations at $0^{\circ}, 45^{\circ}, 90^{\circ}$, and $135^{\circ}$ in the plane of the image), and the fluorescence is detected through a polarizer with the same orientation as the excitation light in the band $515-575 \mathrm{~nm}$, so that the fluorescence intensity depends on the orientation of the LC. The director fields are reconstructed from the experimental data with the help of a simulated annealing algorithm $[19,21]$. In experimental images, the director is shown with streamlines which point along the in-plane projection of the director and are not shown where the director is mostly perpendicular to the plane. The positions of the point defects are identified from the experimental data and marked with color-coded spheres indicating their degree. Gray rods are added between the defects to aid the visualization of their $3 \mathrm{D}$ arrangement.

\section{ACHIRAL POINT DEFECTS}

As we describe now, the generic structure around a point defect involves regions where the twist $\mathbf{n} \cdot \nabla \times \mathbf{n}$ is both positive and negative. This frustrates the preference for a single sense of twist and makes such generic point defects energetically disfavorable in chiral materials.

We represent the director field as $\mathbf{n}=\mathbf{m} /|\mathbf{m}|$ where $\mathbf{m}$ is a vector field with isolated zeros at each of the point defects. Since $\mathbf{n} \cdot \nabla \times \mathbf{n}=(\mathbf{m} \cdot \nabla \times \mathbf{m}) /|\mathbf{m}|^{2}$, the sense of twist in the director field is the same as that in $\mathbf{m}$. We call a defect (zero in $\mathbf{m}$ ) chiral if it is surrounded by a region in which the vector field is twisted of a single handedness, otherwise, we call it achiral. The twist is frustrated at the defects-if $\mathbf{m}=\mathbf{0}$, then also $\mathbf{m} \cdot \nabla \times \mathbf{m}=0$ - so that it is nongeneric for a defect to be chiral. To explain the frustration, at the level of a structural classification the zeros in $\mathbf{m}$ are locally equivalent to a polynomial form and in the generic case to a linear vector field. If the curl is nonzero, then the twist will be a linear function and the zero is not chiral; the twist changes sign across a planar surface passing through the defect. An example is the vector field $\mathbf{m}=x \mathbf{e}_{x}-z \mathbf{e}_{y}+y \mathbf{e}_{z}$ for which the twist is $\mathbf{m} \cdot \nabla \times \mathbf{m}=2 x$. We provide an illustration of this achiral point defect in Fig. 3. Its structural form provides a good local description of the defects that arise close to the droplet surface in experiments, as we describe further in Sec. VI.

We show in Fig. 4(a) the result of a typical simulation of a weakly chiral droplet $(N<1)$. The texture is visualized through a Pontryagin-Thom surface [41], which shows the set of points where the director is horizontal $\left(n_{z}=0\right)$ and colored according to the horizontal orientation. The most noticeable change from the radial profile of a nematic droplet is the displacement of the defect from the center towards the surface. The displacement can be understood

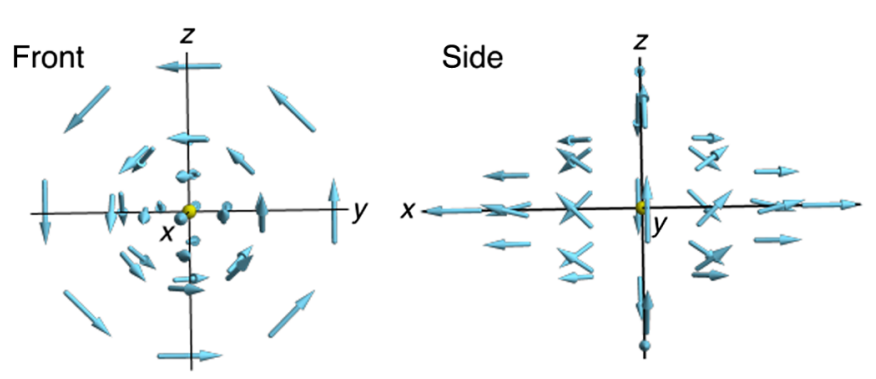

FIG. 3. Two views, "front" and "side," of the local structure of the achiral point defect $\mathbf{m}=x \mathbf{e}_{x}-z \mathbf{e}_{y}+y \mathbf{e}_{z}$, where the twist changes sign across the $x=0$ plane. The $x$ axis exhibits the local structure of a double-twist cylinder, left handed for $x>0$ and right handed for $x<0$.

by looking at the twist throughout the droplet, which we show in Fig. 4(b); the defect sits on a surface that separates the droplet into regions with opposite handedness. There is then an energetic drive to expand the region with the correct handedness (blue) at the expense of that with the wrong sense of twist (red), and the balance of this with the increased elastic distortion determines the position of the point defect. The broken spherical symmetry from the displacement of the defect defines an axis through the droplet, which the chiral distortion of the director field turns into the axis of a double twist cylinder; cf. the local structure around the defect shown in Fig. 3.

In simulations, increasing the value of $N$ but still keeping it small continues this trend; the defect moves further towards the surface to reduce the size of the region with the wrong handedness, and the axis it defines becomes increasingly recognizable as a double-twist cylinder. However, this behavior does not continue for larger values of $N$, and for values of $N \gtrsim 2$, the defect starts to move away from the surface again as the director field restructures continuously to the form shown in Figs. 4(c) and 4(d). These simulation results may be compared with the experimental images of Figs. 4(e) and 4(f) showing a cholesteric droplet containing a single off-center-located point defect. By eye, the comparison is excellent. From simulations, we find that the twist is uniformly right handed around the point defect, meaning that it is chiral and fundamentally different from its initial radial structure. We describe this in Secs. V and VI.

The transition observed here between the states shown in Figs. 4(a) and 4(c) is reminiscent of a transition between radial and hyperbolic point defects observed in nematic droplets with unequal elastic constants [50]. There, the transition is precipitated by a change in the elastic constants with temperature that sees the radial hedgehog become energetically unfavorable relative to the hyperbolic one when the splay elastic constant is sufficiently large compared to twist and bend [51,52]. In our case, the "twist instability" is driven by the explicit chiral coupling, even without elastic anisotropy. This different energetic drive also leads to different phenomenology where the achiral 


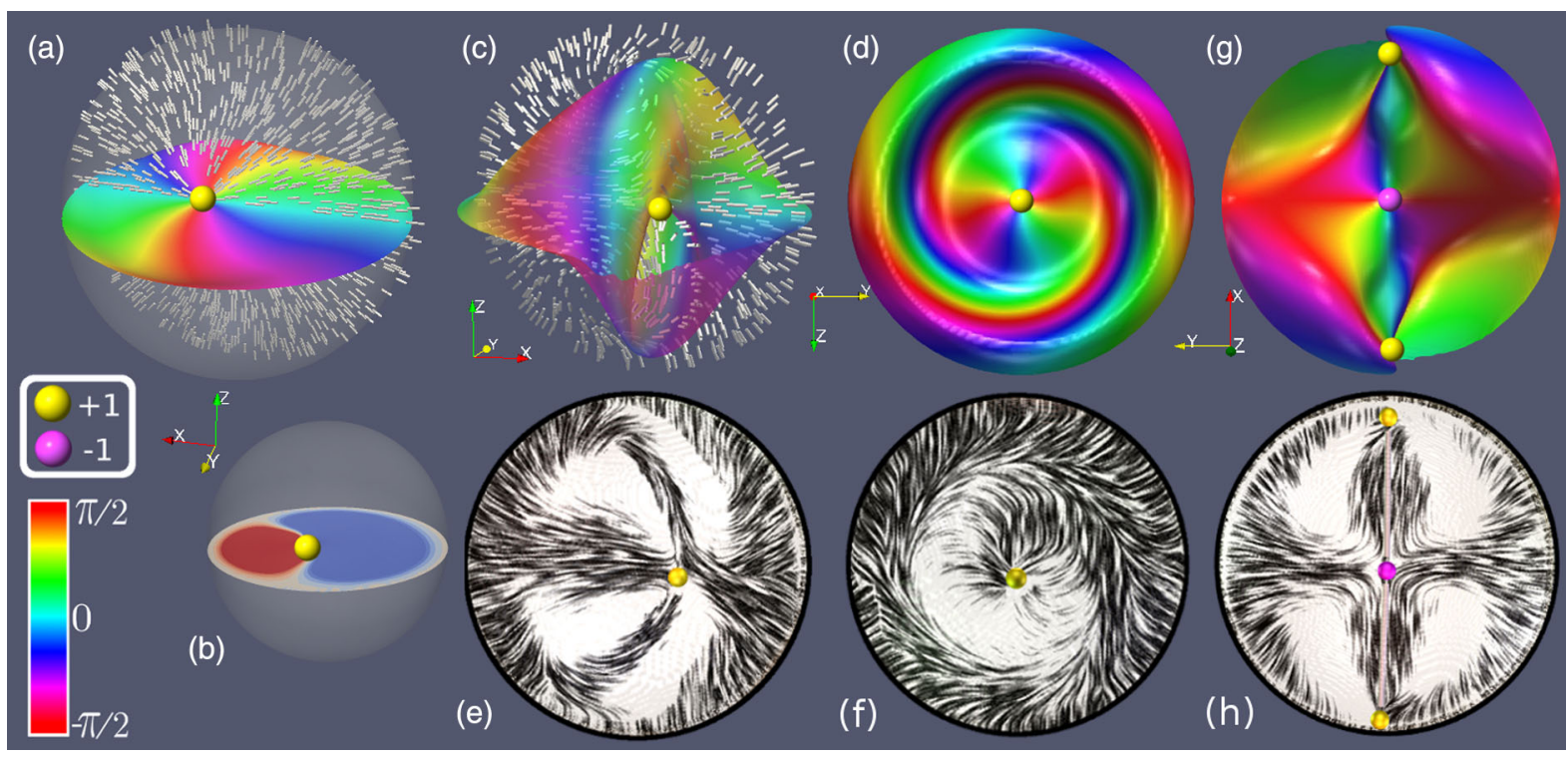

FIG. 4. (a) Generic achiral defect with topological charge +1 obtained for small values of $N$. The surface is the locus $n_{z}=0$ (Pontryagin-Thom surface) with color indicating the angle between the $x$ and $y$ components of the director. (b) The twist on a midplane $x y$ slice showing the reversal of twist at the location of the defect; the left-handed region is shown in red and the right-handed region in blue. (c) $n_{z}=0$ and (d) $n_{x}=0$ surfaces for a chiral defect with Morse index 2. In (c), the director is also shown on a midplane $x z$ slice for added clarity. In (d), the viewing direction is along $x$. Corresponding experimental observations of this defect are shown in (e) and (f). (g) Chiral defect with Morse index 1 and topological charge -1 (central defect); viewing direction along $z$. Two achiral charge +1 defects reside close to the droplet surface. (h) An experimental observation of the same defect. In all panels, defects are highlighted and color coded according to their charge (legend). Panels (e) and (f) reprinted with permission from G. Posnjak, Topological Formations in Chiral Nematic Droplets (2018). Copyright (2018), Springer. Panel (h) reprinted with permission from G. Posnjak et al., Nat. Commun. 8, 14594 (2017). Copyright (2017), the authors.

"radial" defect is expelled from the droplet interior until it sits near the surface.

\section{CHIRAL POINT DEFECTS}

Generic linear vector fields fail to be chiral because their curl is nonzero; as we argue in the previous section, this nonzero curl leads inevitably to the handedness reversing across some surface passing through the defect. Therefore, if the defect is to have any chance of being chiral, these leading-order linear terms must be irrotational. Consequently, they can be written as the gradient of a function $\phi$ with an isolated critical point. As the twist of a gradient field vanishes identically, this leads to the curious situation where the chirality of a cholesteric point defect comes from the subleading terms in its Taylor series. To summarize, the local form for a chiral zero is

$$
\mathbf{m}=\nabla \phi+\mathbf{m}_{c},
$$

where $\phi$ has an isolated critical point at the origin, and $\mathbf{m}_{c}$ is of higher order in a Taylor series. As alluded to, the structure of the director field is then unexpectedly close to that of a smectic, namely, normal to the level sets of $\phi$. The type of critical point in $\phi$ is a label for the chiral zero that refines the topological charge. In the generic case, the critical point is given by one of four quadratic functions referred to as Morse type, as we describe in Sec. II A.

According to these general considerations, the generic chiral defect with topological charge +1 and Morse index 2 is described by the local form

$$
\mathbf{m}=a x \mathbf{e}_{x}-(y-q x z) \mathbf{e}_{y}-(z+q x y) \mathbf{e}_{z},
$$

the linear terms coming from the Morse function and the quadratic terms comprising the higher-order $\mathbf{m}_{c}$. The twist is $\mathbf{m} \cdot \nabla \times \mathbf{m}=-q\left(2 a x^{2}+y^{2}+z^{2}\right)$ giving a uniformly right-handed texture everywhere except at the defect itself. Here, $a>0$ is dimensionless and $q$ has dimensions of an inverse length. For the particular case $a=2, \mathbf{n} \cdot \nabla \times \mathbf{n} \approx$ $-q$ suggesting that we should take $q=q_{0}$ up to an $O(1)$ factor. Changing the sign of the vector field (6) gives the local form of a generic chiral defect of topological charge -1 and Morse index 1. The local structure of the vector field (6) is shown in Fig. 2, while in Figs. 4(c) and 4(d) we show the results of simulations initialized using Eq. (6) or its negative $(g)$ throughout the central portion of the droplet and a radial profile outside. The correspondence with experimental observations [19-21] is striking. These are shown in Figs. 4(e), 4(f), and 4(h). We defer discussion of the structure of the twist throughout these droplets to the following section. 
The radial hedgehog $\mathbf{m}=x \mathbf{e}_{x}+y \mathbf{e}_{y}+z \mathbf{e}_{z}$ corresponds to the gradient field of the function $\phi=\frac{1}{2}\left(x^{2}+y^{2}+z^{2}\right)$, with Morse index 0 , and is the observed configuration for nematic droplets (with equal elastic constants). However, this structure is not preserved in a cholesteric droplet and exhibits fundamental frustration with a state of uniform twist; there is no choice of higher-order term $\mathbf{m}_{c}$ that makes the radial hedgehog chiral. This is a topological result that follows from a theorem of Eliashberg and Thurston [53], the Reeb stability theorem for confoliations, which we describe informally. It is based on the property that the level sets of $\phi$ are spheres. Consider any such level set and separate it into two hemispheres with common boundary an equator. The twist over each hemisphere can be related to the nature of the director field on its boundary. This relation is based on the properties of integral curves of the plane field orthogonal to $\mathbf{n}$, i.e., curves whose tangent is always perpendicular to the director. When the twist vanishes, the director is the normal to the level sets of $\phi$, and any curve lying in such a surface is integral. This applies in particular to the boundary of any disk. When the twist is nonzero, the director is no longer perpendicular to the boundary. In this case, the boundary can be lifted-pushed up or down along the surface normal direction-to create an integral curve. This curve is not closed; its end points have a vertical displacement between them whose magnitude is related to the nonvanishing of the twist [53]. An illustration is shown in Fig. 5. This description relates the twist to a holonomy, an association of closed loops in a surface to displacements along the surface normal direction. The holonomy can be viewed as a connection on a line or circle bundle over the surface, whose curvature yields the twist [53].
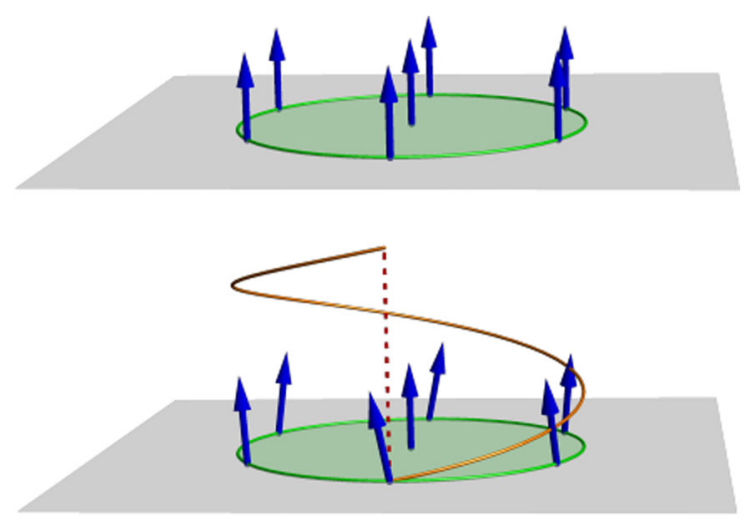

FIG. 5. Schematic illustration of the twist as a holonomy. (Top) When the twist vanishes, the director coincides with the normal to a surface and is perpendicular to the boundary of any disk lying in the surface. (Bottom) When the twist is nonzero and the director is no longer the surface normal, the disk boundary can be lifted along the surface normal to give an open curve, again perpendicular to the director. The distance between the end points gives a measure of the twist.
Importantly, if the twist is right handed, then the displacement is along the positive normal direction (see Fig. 5), while if it is left handed, the displacement will be in the negative direction. Applying this to the separation of any spherical level set of a radial hedgehog into two hemispheres, we see that the displacement around the boundary of each hemisphere is equal and opposite, since the two boundaries are the same equator but traversed in opposite directions. As a result, if the twist is right handed in one hemisphere, it will be left handed in (at least part of) the other, and in equal measure. We see that radial hedgehogs are incompatible with the preferred handedness of cholesterics and engender a topological frustration of twist in the director field.

\section{DROPLET SURFACE AND BOUNDARY LAYER}

Precisely the same argument applies to a boundary layer at the droplet surface where the director becomes radial to match the boundary conditions. By the same theorem of Eliashberg and Thurston, the twist cannot be uniformly right handed, and by necessity there is some region of reversed twist close to the surface. To elaborate briefly on Eliashberg and Thurston's theorem, we should clarify that what they consider are confoliations-nonsingular vector fields with non-negative twist. The result they prove establishes that if the vector field is normal to a spherical surface and has non-negative twist everywhere in a neighborhood of that surface, then the twist must vanish identically, and the vector field is the normal to a family of spherical surfaces. While it is mathematically natural to consider a restriction that the twist should never be negative, there is no such physical barrier in chiral materials but only an energetic cost. For physical systems in which nothing fundamentally prevents regions of reversed handedness, the natural restatement of Eliashberg and Thurston's theorem is as we present it: If the material contains a spherical surface that the director field is transverse to, then either the twist vanishes identically or there are regions of both handedness in any neighborhood of that surface. The former possibility is highly nongeneric in a chiral material, and we typically omit its explicit mention for expediency.

This version of the result applies to the boundary layer near the droplet surface and also to the local neighborhood of any radial point defect, as we describe in the previous section. Furthermore, it provides a topological demarcation of the surface boundary layer in a chiral droplet, namely, the region near the surface in which the director remains transverse to the family of concentric spheres extending in from the boundary itself.

To expand a little on the topological character of the boundary layer behavior, note that the heliconical director

$$
\mathbf{n}=\cos \theta(z) \mathbf{e}_{z}+\sin \theta(z)\left[\cos q z \mathbf{e}_{x}+\sin q z \mathbf{e}_{y}\right]
$$

describes an interpolation from normal surface anchoring $\theta(0)=0$ on the plane $z=0$ to a chiral texture (the twist 
is $-q \sin ^{2} \theta$ ) away from the surface. This example establishes that there can be no local frustration of the twist by normal surface anchoring (except, potentially, for energetic reasons). The frustration comes from the global topology of the surface. Interestingly, it is peculiar to spherical droplets; if the liquid crystal is instead confined to a handlebody droplet of any positive genus - the number of holes or handles through the droplet-there is no topological obstruction to a uniform sense of twist (handedness) in the boundary layer near the surface [53].

These general considerations assert that there must be some region of reversed twist close to the spherical droplet surface but do not say anything about its actual form. We find from our numerical simulations that two situations arise, exemplified by the chiral Morse index 1 and 2 defects. In the case of the Morse index 2 defect [Fig. 4(c)], the region of reversed twist takes the form of a nonsingular ring, as shown in Fig. 6(a). The twist is right handed throughout the interior of the droplet but becomes left handed in a ring localized near the droplet surface. The ring lies in a vertical $y-z$ plane at the $x$ location of the defect (slightly displaced from the center). This region of reversed twist is a novel nonsingular topological soliton. Its existence is protected by topology, and the energetic cost of having the wrong handedness leads to it being strongly spatially localized. The structure of the director field across the soliton is shown in Figs. 6(b) and 6(c).

In all other cases, the chiral point defects in the droplet interior are encaged by an arrangement of charge +1 defects localized close to the droplet surface. These "surface" defects are achiral and demarcate a localized region between themselves and the droplet surface in which the sense of twist reverses. An example of the Morse index 1 defect is shown in Fig. 6(d), with the structure of the director close to the achiral defect given in Fig. 6(e). The topological argument for twist reversal near the boundary provides an explanation for the presence of such defects localized close to the surface, although we should emphasize that they are not topologically bound to the surface and so held there only by an energetic barrier.

Regions of opposite twist localized near point defects close to surfaces with normal anchoring have recently been observed in experiments and numerical simulations [54], although in those, the surface is the flat plane of a glass slide as opposed to being spherical. It is only spherical surfaces that have a topological requirement for regions of reversed twist. The appearance of regions of opposite twist in situations where it is not topologically required is then an energetic phenomenon.

The problem of classifying vector fields around a surface across which the twist changes sign was first studied by Martinet [55] and later extended by Zhitomirskiu [56], who showed that the director in a neighborhood of a generic defect with topological charge +1 is [56]

$$
\mathbf{m}=2 x \mathbf{e}_{x}-z \mathbf{e}_{y}+y \mathbf{e}_{z} .
$$

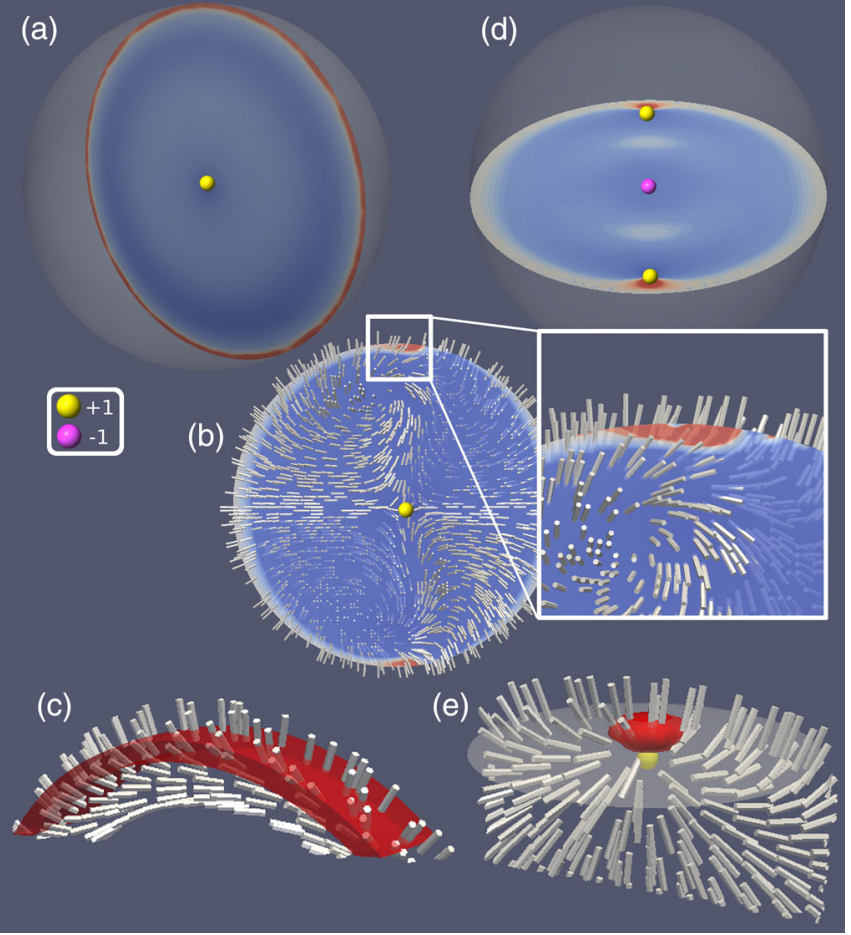

FIG. 6. Boundary layer and reversal of twist. (a) Chiral structure of a droplet containing a Morse index 2 defect showing the twist on a vertical $y-z$ plane passing through the defect. The twist is uniformly right handed (blue) around the central defect, but there is a localized ring of twist reversal (red) near the surface. (b) Structure of the director field on a vertical $x-z$ plane, with an inset showing the director close to the ring of reversed twist. (c) Structure of the director close to the ring of reversed twist, which is shown in red. (d) The twist on a midplane ( $z$ slice) through a droplet containing a Morse index 1 chiral defect [Fig. 4(g)]. The blue region is right handed; the red regions between the surface point defects and the surface itself are left handed. This structure is common to all surface point defects. Note that the twist is uniformly right handed around the central charge -1 defect. (e) Structure of the director in a region around an achiral defect anchored to the surface of the droplet (white surface). The region of reversed handedness is shown in red.

In this form, the twist changes sign as we cross the plane $x=0$ so that the defect is achiral, and the local structure is as shown in Fig. 3. Introducing higher-order terms into $\mathbf{m}$ controls the shape of the interface between the regions of opposite handedness. For instance, adding a term $q x\left(-y \mathbf{e}_{y}+z \mathbf{e}_{z}\right)$ leads to the twist vanishing on the parabolic surface $4 x=q\left(y^{2}+z^{2}\right)$. The region of incorrect handedness is the interior $4 x>q\left(y^{2}+z^{2}\right)$ of this paraboloid whose shape is similar to that observed in simulations [Fig. 6(e)].

\section{SINGULARITY THEORY AND DEFECTS OF HIGHER DEGREE}

Morse critical points have topological charge \pm 1 , so they cannot describe the defects with higher charge $(-2,-3)$ 
observed experimentally [20]. The higher charge defects are still described by the form (5) but with a degenerate critical point in the function $\phi$. The classification of degenerate critical points is significantly more involved than that of Morse critical points; here, we follow Arnold's classification [57]. Although it is natural to expect that the simplest degenerate critical points will appear first, this is not the case, essentially because the topological charge of the gradient field is not central to the classification of degenerate critical points. As we show, the experimentally observed higher-charge defects are captured by the elliptic umbilic, or $D_{4}^{-}$, singularity (charge -2 ) and the $T_{4,4,4}$ singularity (charge $-3)$. Moreover, we show that the topological molecules that have been observed are consistent with the unfoldings of these and higher-charge singularities.

An initial determination of degenerate critical points was given by René Thom in his study of structural stability and morphogenesis [35]. Thom identified the most generic singularities - those that require the tuning of the fewest parameters - and described the families in which they arise. These are the so-called elementary catastrophes: the fold, cusp, swallowtail, elliptic umbilic, and hyperbolic umbilic. They were quickly identified in optical caustics [36], where they arise in exactly the manner that Thom prescribed, as well as a host of other examples in the physical, biological, and social sciences and economics [37].

A systematic study of degenerate critical points can be developed from their local algebra: loosely, the linearly independent polynomials of lower order than anything appearing in the gradient field; more formally, the quotient of the ring of Taylor series $\mathbb{R}[x, y, z]$ by the gradient ideal $\left\langle\partial_{x} \phi, \partial_{y} \phi, \partial_{z} \phi\right\rangle$. The dimension of this local algebra is called the multiplicity of the singularity: It is the number of nondegenerate critical points that it splits into (as a complex polynomial) under a generic perturbation. Said differently, the multiplicity is the number of nondegenerate critical points that simultaneously coalesce in forming the singularity. It is typical to represent a singularity by a standard polynomial called its normal form. The simplest degenerate critical points have unique normal forms; however, the more degenerate ones have moduli, or free continuous parameters. The number of moduli appearing in the normal form is called the modality of the singularity. The singularities without moduli known as the simple singularities were completely listed by Arnold in his celebrated $A D E$ classification [58]; there are two infinite families and three exceptional cases:

$$
\begin{aligned}
A_{k}^{ \pm} & : \pm x^{k+1}(k \geq 1), \\
D_{k}^{ \pm} & : x^{2} y \pm y^{k-1}(k \geq 4), \\
E_{6} & : x^{3} \pm y^{4}, \\
E_{7} & : x^{3}+x y^{3}, \\
E_{8} & : x^{3}+y^{5} .
\end{aligned}
$$

In three dimensions, these minimal models should be supplemented by quadratic terms in the remaining variables, i.e., $y$ and $z$ for the $A_{k}^{ \pm}$singularities, and only $z$ for the others. For instance, for the $A_{2}$ singularity we might write $\pm x^{3}+y^{2}+z^{2}$. The subscript indicates the multiplicity of the singularity.

The addition of less degenerate terms to the normal form of any singularity results in a simplification of the singularity known as an unfolding and usually breaking it into multiple critical points of lower multiplicity. The number of independent terms in a complete unfolding is called the codimension and can be thought of as the number of parameters that need to be tuned in order to create the degenerate critical point. For example, for the $A_{k}$ singularity, a complete unfolding consists of the monomials $x^{n}$ for $n=1, \ldots, k-1$ and the codimension is $k-1$. In general, the multiplicity $\mu$, codimension $c$, and modality $m$ are related by $\mu=c+m+1$.

Among the simple singularities (9), the only models for defects with topological charge -2 are furnished by the classes $D_{2 k}^{-}$; all the other simple singularities have charge 0 or \pm 1 . Therefore, the simplest model for a point defect of topological charge -2 comes from the simple singularity $D_{4}^{-}$, which we write as

$$
\phi=\kappa\left(x^{2} y-\frac{1}{3} y^{3}\right)+\frac{1}{2} z^{2},
$$

where $\kappa$ is a constant with dimensions of an inverse length. The structure of this critical point is shown in Fig. 7(a). Its gradient vector field can be made chiral in a neighborhood of the origin by adding the higher-order term

$$
\mathbf{m}_{c}=q\left[-\kappa z\left(x^{2}-y^{2}\right) \mathbf{e}_{x}+2 \kappa x y z \mathbf{e}_{y}\right]-q^{2} z^{3}\left[-y \mathbf{e}_{x}+x \mathbf{e}_{y}\right] .
$$

The correspondence between this local model and experimental observations [20,21] is striking [Figs. 8(a) and 8(b)], although we find in our simplified director-based (a)

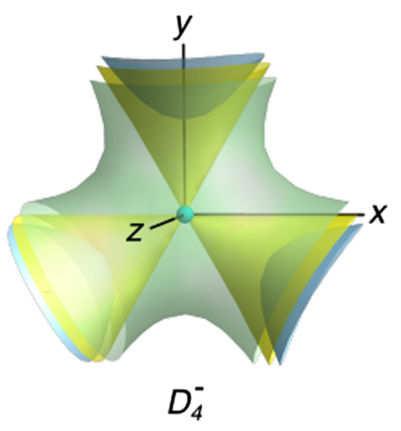

(b)

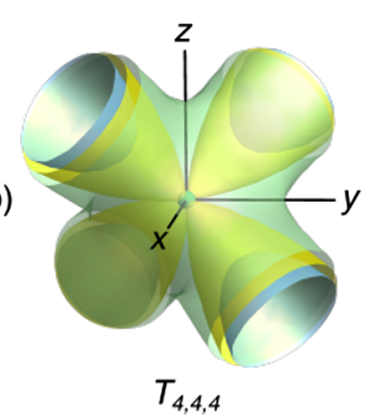

FIG. 7. Local structure of the critical points (a) $D_{4}^{-}$and (b) $T_{4,4,4}$, whose gradient fields provide the local structure for chiral point defects with topological charge -2 and -3 , respectively. 


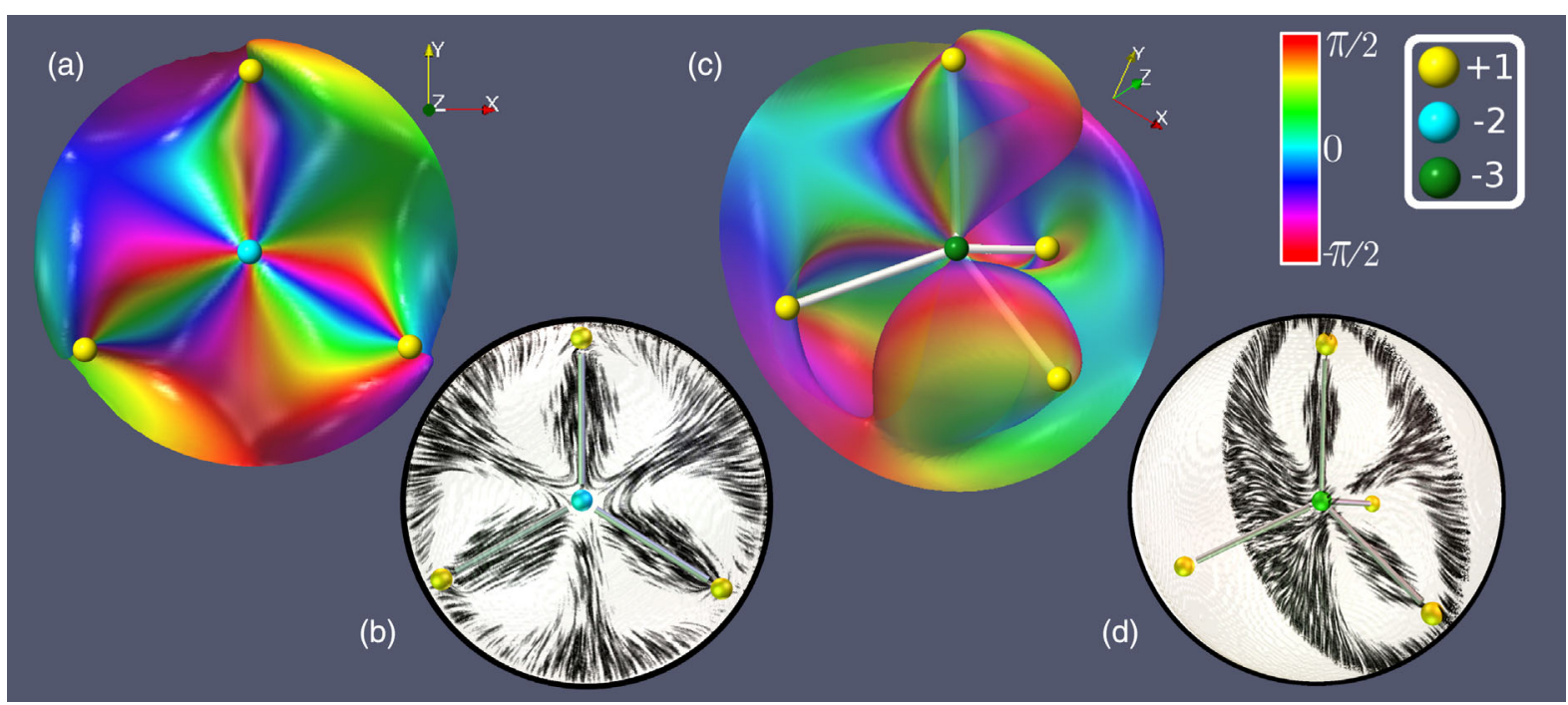

FIG. 8. (a) Point defect with topological charge -2 associated with the $D_{4}^{-}$singularity. The surface is the locus $n_{z}=0$ (PontryaginThom surface), with color indicating the angle between the $x$ and $y$ components of the director. (b) Experimental realization of the charge -2 defect with superimposed director field in the $z=0$ droplet midplane. (c) Point defect with topological charge -3 associated with the $T_{4,4,4}$ singularity ("bonds" between defects added for visual clarity). (d) Corresponding experimental image of a droplet containing a charge -3 defect. In all panels, defects are highlighted and color coded according to their degree. Panel (b) reprinted with permission from G.Posnjak et al., Nat. Commun. 8, 14594 (2017). Copyright (2017), the authors.

simulations that the defect is unstable and breaks apart into two charge -1 defects, each with the Morse-type local structure described above. This points to the importance of defect core structure, or elastic anisotropy, for stability in physical systems.

The experimentally observed defect with topological charge -3 [20,21] [Fig. 8(d)] is reproduced by the unimodal singularity $T_{4,4,4}$, which we write

$$
\phi=\operatorname{axyz}+\frac{1}{4}\left(x^{4}+y^{4}+z^{4}\right)
$$

where the modulus $a$ has dimensions of length. Its local structure is shown in Fig. 7(b). $T_{4,4,4}$ is a codimension 9 singularity with multiplicity 11 characteristics which convey its high complexity. In addition to the origin where there is an isolated zero of topological charge -3 , the gradient vector field has isolated zeros of charge +1 (and Morse index 0 ) at the four points $a(1,-1,1), a(-1,1,1)$, $a(1,1,-1), a(-1,-1,-1)$ corresponding to the vertices of a tetrahedron. This suggests that the modulus $a$ should take a value of $R / \sqrt{3}$ up to an $O(1)$ factor, so that these defects sit near the surface of the droplet, as we observe experimentally. The gradient field of Eq. (12) can be perturbed into a chiral point defect via a generic method that we describe in the following section. Simulations initialized with the $T_{4,4,4}$ singularity produce a numerically stable charge -3 point defect surrounded by four tetrahedrally arranged surface defects in excellent agreement with the experiment [Fig. 8(c)].

\section{A. Unfoldings and topological molecules}

In addition to droplets containing individual chiral point defects, experiments also reveal a variety of multiple-defect structures [20]. Unlike nematic droplets, these multidefect cholesteric textures are metastable, and the defects do not annihilate pairwise to obtain a minimal number. Moreover, the arrangements appear far from random, exhibiting definite structure and symmetry, leading to them being dubbed as "constellations" and topological molecules. As we show in this section, the unfoldings of singularities provide a natural framework for these topological molecules, with excellent agreement with experiment. Subsequently, in Sec. IX, we show that the geometric structure of cholesterics, and in particular, their degeneracies known as $\lambda$ lines or umbilics, serve to furnish the chemical bonds of these topological molecules.

The unfoldings of singularities provide systematic descriptions of how degenerate critical points can break apart into simpler pieces or how generic critical points coalesce to form degenerate ones. In the present context, these unfoldings provide models for the combination and splitting of chiral point defects. For instance, the simplest description of the annihilation of two chiral defects with opposite topological charge is given by the unfolding of the $A_{2}$ singularity

$$
\phi=\frac{\kappa}{3} x^{3}+\frac{1}{2} y^{2}-\frac{1}{2} z^{2}+c_{1} x,
$$

where $\kappa$ is a constant with dimensions of an inverse length, and $c_{1}$ is a parameter of the unfolding with dimensions of 
length. When $c_{1} / \kappa$ is positive, there are no critical points, while when it is negative, there are isolated Morse critical points at $\left( \pm\left|c_{1} / \kappa\right|^{1 / 2}, 0,0\right)$ with Morse indices $1(+)$ and 2 (-). Note that in the way we write the $A_{2}$ singularity (13), the Morse terms appear with opposite signs, i.e., $y^{2}-z^{2}$. This structure ensures that after unfolding, the two Morsetype defects are both chiral; if these Morse terms are both given the same sign $\left[ \pm\left(y^{2}+z^{2}\right)\right]$, then one of the two critical points that the singularity splits into will have Morse index $0(+)$ or $3(-)$ and, hence, will not be chiral.

The unfolding of the $D_{4}^{-}$singularity provides a description of the splitting of a charge -2 chiral defect, or merging of two point defects with the same charge $(-1)$,

$\phi=\kappa\left(x^{2} y-\frac{1}{3} y^{3}\right)+\frac{1}{2} z^{2}+c_{1} x+c_{2} y+\frac{c_{3}}{2}\left(x^{2}+y^{2}\right)$.

The $c_{3}$ term is isotropic and does not alter the symmetry of the defect; it is therefore natural to consider it separately, and we start with the case $c_{3}=0$. Critical points of $\phi$ are given by $z=0$ and (using a compact complex notation)

$$
\partial_{y} \phi+i \partial_{x} \phi=\kappa(x+i y)^{2}+c_{2}+i c_{1}=0 .
$$

There are two Morse critical points (both of Morse index 1) with locations (assuming $\kappa>0$ )

$$
x+i y= \pm \frac{\left(c_{1}^{2}+c_{2}^{2}\right)^{1 / 4}}{\sqrt{\kappa}} e^{i(\alpha+\pi) / 2},
$$

where $\alpha=\arctan c_{1} / c_{2}$. The splitting is along the $x$ axis when $c_{1}=0$ and $c_{2}$ is negative. This produces a "V-shaped" arrangement of defects within the cholesteric droplet and is strongly reminiscent of the constellations observed experimentally [Figs. 9(a) and 9(b)] [20,21]. The "T-shaped" splitting produced when $c_{2}$ is positive $\left(c_{1}\right.$ still zero) has not been observed in experiments, but it is readily produced numerically [Fig. 9(c)].

Returning to the $c_{3}$ term, consider now the case where only it is nonzero. Then, the $D_{4}^{-}$singularity splits into four critical points, again all lying in the $z=0$ plane: one at the origin of Morse index 2 and three of Morse index 1 at the points

$$
(x, y)=\left(0, \frac{c_{3}}{\kappa}\right), \quad\left( \pm \frac{\sqrt{3} c_{3}}{2 \kappa}, \frac{c_{3}}{2 \kappa}\right) .
$$

This is shown in Fig. 9(d).

The unfoldings of the $T_{4,4,4}$ singularity and the resultant topological molecules are significantly more involved mathematically due to the high codimension and multiplicity. For this reason, we restrict our presentation to consideration of particular unfoldings most directly

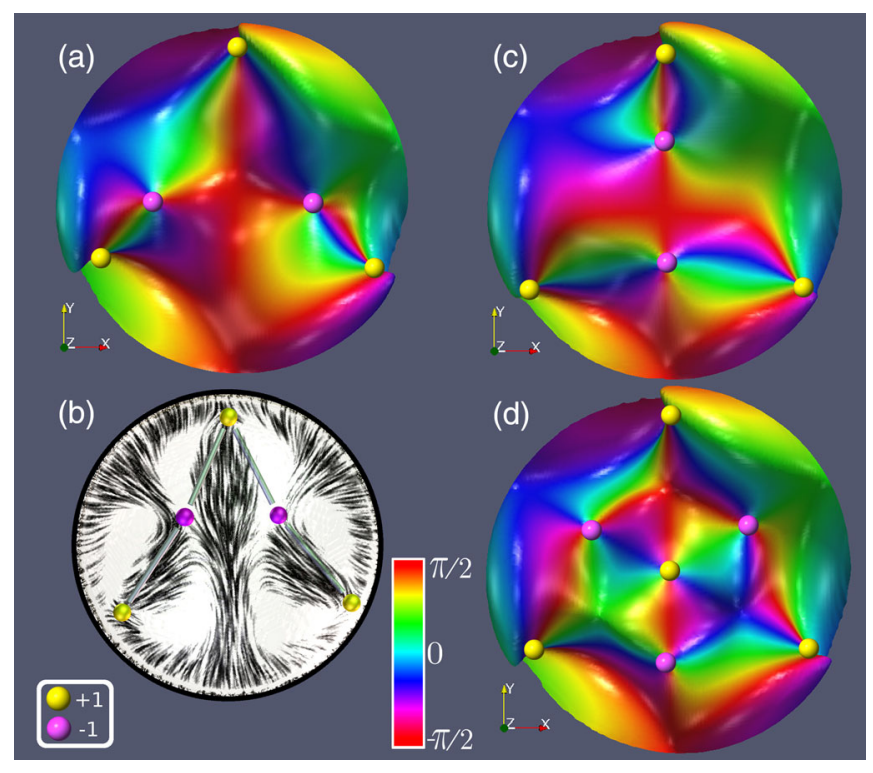

FIG. 9. Unfoldings of the $D_{4}^{-}$singularity. (a) A splitting of the charge -2 defect into two Morse index 1 defects producing a V-shaped constellation closely similar to textures observed experimentally. (b) An example of such an experimental configuration with the director field in the $z=0$ droplet midplane superimposed. (c) By tuning the unfolding, the splitting can also produce a $\mathrm{T}$-shaped constellation, which remains stable numerically but has not been observed in experiments. (d) The isotropic term in the unfolding splits the $D_{4}^{-}$singularity into four Morse critical points without breaking the symmetry of the droplet. Again, this is stable numerically but has not been observed in experiments. Panel (b) reprinted with permission from G. Posnjak et al., Nat. Commun. 8, 14594 (2017). Copyright (2017), the authors.

pertinent to the observed topological molecules. A basis for the local algebra of the $T_{4,4,4}$ singularity can be taken to be the 11 monomials

$$
1, x, y, z, x^{2}, y^{2}, z^{2}, x^{3}, y^{3}, z^{3}, x y z
$$

Note that, e.g., $x^{3} \sim-a y z$ as elements of the local algebra, so that we could replace $x^{3}, y^{3}$, and $z^{3}$ by $y z, z x$, and $x y$ if desired. The constant obviously does not change the nature of the critical point, while the term $x y z$ acts only to change the value of the modulus. The remaining nine terms are associated with the unfoldings, of which we consider two particular cases pointed to by symmetry. We describe each of these in terms of the tetrahedral environment of the $T_{4,4,4}$ singularity. In the first, the threefold symmetry about an axis connecting a vertex and face center of the tetrahedron is preserved: We refer to this as the vertex-face unfolding. In the second, we preserve the twofold rotational symmetry about the $z$ axis: As this connects two edges of the tetrahedron, we refer to it as the edge-edge unfolding. These two geometries are shown schematically in Figs. 10(a) and 10(d). 
The analysis of the vertex-face unfolding is naturally given in terms of the adapted coordinates

$$
\begin{aligned}
u & =\frac{1}{\sqrt{2}}(x-y), \\
v & =\frac{1}{\sqrt{6}}(x+y-2 z), \\
w & =\frac{1}{\sqrt{3}}(x+y+z),
\end{aligned}
$$

in which the $T_{4,4,4}$ singularity takes the form

$$
\begin{aligned}
\phi= & \frac{a}{\sqrt{6}}\left(u^{2} v-\frac{1}{3} v^{3}\right)-\frac{a}{2 \sqrt{3}}\left(u^{2}+v^{2}\right) w+\frac{a}{3 \sqrt{3}} w^{3} \\
& +\frac{1}{8}\left(u^{2}+v^{2}\right)^{2}+\frac{1}{12} w^{4}+\frac{1}{2}\left(u^{2}+v^{2}\right) w^{2} \\
& +\frac{1}{\sqrt{2}}\left(u^{2} v-\frac{1}{3} v^{3}\right) w .
\end{aligned}
$$

We consider an unfolding that preserves the threefold symmetry about the $w$ axis given by the addition of the terms (terms in $w^{2}$ and $w^{3}$ are omitted for simplicity)

$$
c_{1} w+\frac{c_{2}}{2}\left(u^{2}+v^{2}\right)
$$

There are critical points with $u=v=0$ and $w$ given by the roots of the cubic

$$
c_{1}+\frac{a}{\sqrt{3}} w^{2}+\frac{1}{3} w^{3}
$$

When $c_{1}=0$, these reduce to $w=0$ (double root) and $w=-\sqrt{3} a$; when $c_{1}>0$, the former disappear and only the latter remains; when $c_{1}<0$, the double root resolves to two simple roots at $w \approx \pm\left(-\sqrt{3} c_{1} / a\right)^{1 / 2}$. We consider only this last case. Expanding about one of the new critical points, which we denote $w=r$, gives

$$
\begin{aligned}
\phi \sim & \frac{c_{2}-a r / \sqrt{3}}{2}\left(u^{2}+v^{2}\right)+\frac{a r}{\sqrt{3}}(w-r)^{2} \\
& +\frac{a}{\sqrt{6}}\left(u^{2} v-\frac{1}{3} v^{3}\right)
\end{aligned}
$$

corresponding to a Morse critical point. Neglecting $c_{2}$, the Morse index is 1 if $r$ is negative and 2 if $r$ is positive. The latter case we may view as an unfolding of the $D_{4}^{-}$ singularity that we recover by setting $c_{2}=a r / \sqrt{3}$. In this case, the initial $T_{4,4,4}$ singularity splits into a Morse index 1 critical point in the direction of the vertex and a $D_{4}^{-}$ singularity in the direction of the face center of the tetrahedron formed by the accompanying charge +1 surface defects.
This picture is confirmed by numerical simulations of the unfolding, which, moreover, produce a charge -2 chiral defect corresponding to the $D_{4}^{-}$singularity that is numerically stable for orders of magnitude longer than the directly initialized defect. An example of such a simulation showing the Pontryagin-Thom surface $n_{w}=0$ is shown in Fig. 10(b) and can be compared with the topological molecule observed experimentally and shown in Fig. 10(c); the agreement is striking.

Turning now to the edge-edge unfolding, we consider the function

$\phi=\operatorname{axyz}+\frac{1}{4}\left(x^{4}+y^{4}+z^{4}\right)+c_{1} z+\frac{c_{2}}{2} z^{2}+\frac{c_{3}}{3} z^{3}+d x y$,

where the parameters $c_{k}$ have dimensions of (length) $)^{4-k}$ and $d$ has dimension (length) ${ }^{2}$. There are critical points with $x=y=0$ and $z$ given by the roots of the cubic $z^{3}+c_{3} z^{2}+c_{2} z+c_{1}$. The number of roots depends on the value of the discriminant

$$
27 c_{1}^{2}-18 c_{1} c_{2} c_{3}+4 c_{1} c_{3}^{3}+4 c_{2}^{3}-c_{2}^{2} c_{3}^{2} .
$$

When this is positive, there is only one root; when it is negative, there are three; when it vanishes, there are two. Expanding about one of the roots (at $z=r$ ) gives the form $\sim(a r+d) x y \pm(z-r)^{2}$, which is of Morse type and Morse index $1(+)$ or $2(-)$, the latter occurring only for the "middle" root when there are three roots. There are additional critical points off axis $(x, y \neq 0)$ unless the parameter $d$ takes the value $d=-a r$, with $r$ corresponding to the middle critical point. It is then of type $X_{9}=T_{2,4,4}$ and has topological charge -1 . We emphasize that this unfolding produces a linear string of three chiral point defects each with topological charge -1 but which are not all equivalent; the two outer defects have a local structure corresponding to the gradient field of a Morse critical point with Morse index 1 , whereas the middle defect has the local structure of the gradient field of an $X_{9}$ singularity. A numerical simulation of this unfolding is shown in Fig. 10(e).

When there is a double root (at $z=r$ ), the function has the form $(a r+d) x y+r(z-r)^{3}$ corresponding to an $A_{2}$ singularity with topological charge 0 , unless $d=-a r$ when it is instead a $T_{3,4,4}$ singularity with charge -2 . Thus, the function

$\phi=\operatorname{axy}(z-r)+\frac{1}{4}\left(x^{4}+y^{4}+z^{4}\right)-\frac{3 r^{2}}{2} z^{2}+2 r^{3} z$

describes, for $r \neq 0$, a splitting of the $T_{4,4,4}$ singularity into a Morse index 1 critical point at $z=-2 r$ and a $T_{3,4,4}$ singularity at $z=r$. A numerical simulation of this unfolding is shown in Fig. 10(e); however, in this case, the charge -2 defect is not stable numerically and splits 


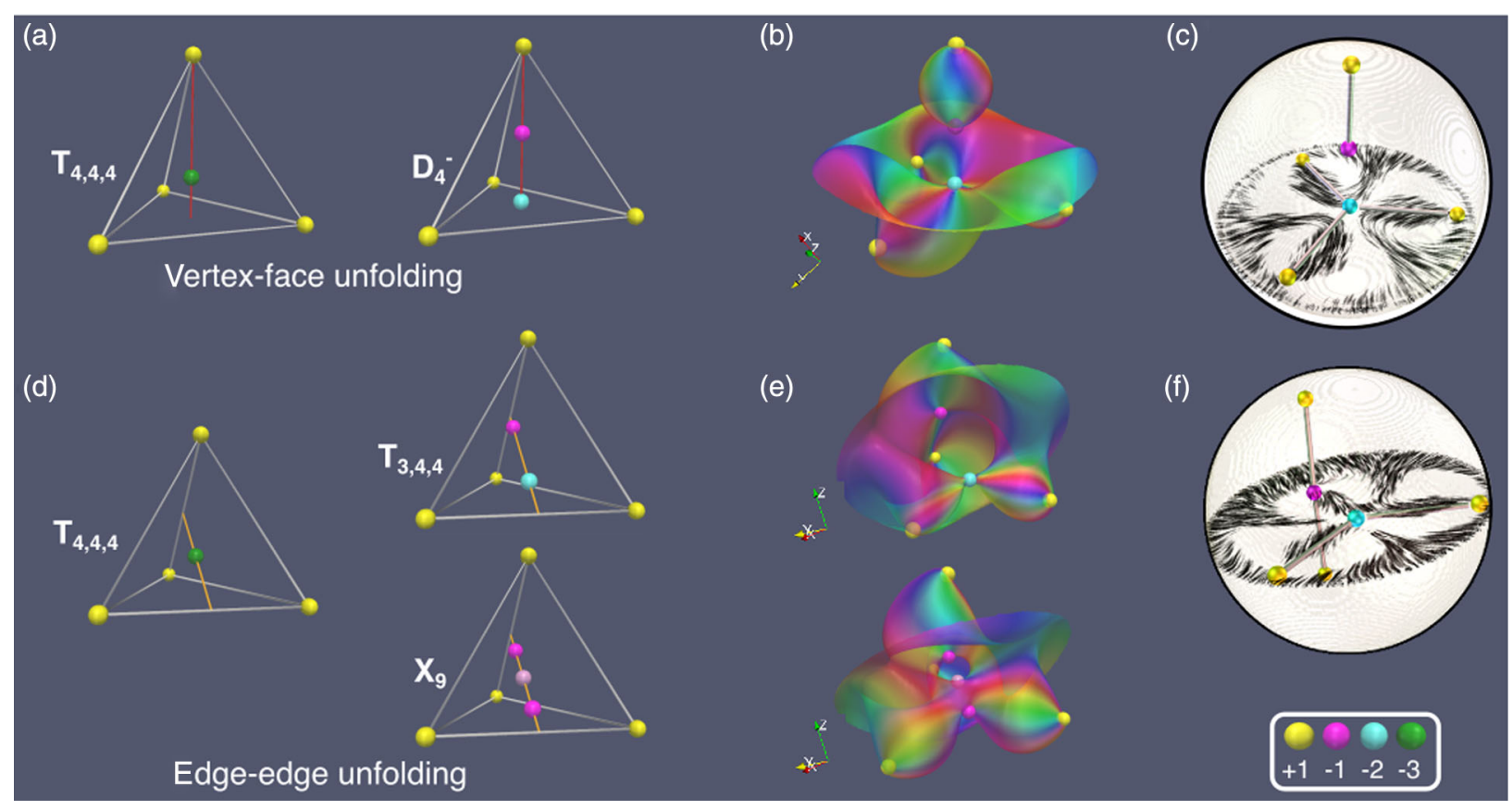

FIG. 10. Unfoldings of the $T_{4,4,4}$ singularity and topological molecules. (a) Schematic of the vertex-face unfolding splitting $T_{4,4,4}$ into a Morse index 1 and $D_{4}^{-}$singularities. (b) Numerical simulation of the unfolding: The charge -2 defect remains stable for long times. (c) Topological molecule observed experimentally corresponding to the vertex-face unfolding. (d) Schematic of the edge-edge unfolding, with splittings of $T_{4,4,4}$ into two Morse index 1 critical points and an $X_{9}$ singularity, with topological charge -1, or into a single Morse critical point and a $T_{3,4,4}$ singularity, with topological charge -2 . (e) Numerical simulation of the unfolding: The charge -2 defect is unstable numerically and is shown here artificially stabilized. (f) Topological molecule observed experimentally corresponding to the edge-edge unfolding. Panel (c) reprinted with permission from G. Posnjak, Topological Formations in Chiral Nematic Droplets (2018). Copyright (2018), Springer.

(transverse to the edge-edge axis) into two Morse index 1 defects. Nonetheless, this configuration of point defects is observed experimentally [Fig. 10(f)] with a stable charge -2 defect, and its structural similarity to the edge-edge unfolding is strongly suggestive. The main implication is that the two charge -2 defects in the observed topological molecules-Figs. 10(c) and 10(f)_correspond to topologically distinct chiral defects, one having the structure of the $D_{4}^{-}$singularity and the other the structure of the $T_{3,4,4}$ singularity.

\section{CURL EIGENFIELDS AND HARMONIC CRITICAL POINTS}

A useful special model for chiral point defects is obtained by taking an extreme limit of the Frank free energy (4) where the twist elastic constant is much larger than both splay and bend, $K_{2} \gg K_{1}, K_{3}$. In this limit, minimizers should pointwise satisfy $\mathbf{n} \cdot \nabla \times \mathbf{n}=-q_{0}$, i.e., have a constant value for the twist. This is attained for directors that derive from curl eigenfields. A curl eigenfield, also known as a Beltrami field, is a vector $\mathbf{m}$ for which $\nabla \times \mathbf{m}=\lambda \mathbf{m}$ for a scalar eigenvalue $\lambda[59,60]$. Setting $\mathbf{n}=\mathbf{m} /|\mathbf{m}|$, we obtain $\mathbf{n} \cdot \nabla \times \mathbf{n}=\lambda$ so that a normalized curl eigenfield with eigenvalue $\lambda=-q_{0}$ provides a special model for chiral textures and their defects reminiscent of that for the blue phases [61] or their helimagnet analogues $[62,63]$.

Curl eigenfields are divergenceless, and in the local form $\mathbf{m}=\nabla \phi+\mathbf{m}_{c}, \phi$ is a harmonic function. The chiral perturbation is also determined by $\phi$; as a formal series, $\mathbf{m}_{c}=\sum_{j} \mathbf{m}_{c}^{(j)}$, we have the recursive equations

$\nabla \times \mathbf{m}_{c}^{(1)}=-q_{0} \nabla \phi, \quad \nabla \times \mathbf{m}_{c}^{(j)}=-q_{0} \mathbf{m}_{c}^{(j-1)}$,

together with $\nabla \cdot \mathbf{m}_{c}^{(j)}=0$. Each term $\mathbf{m}_{c}^{(j)}$ is determined up to the addition of a harmonic gradient. Thus, the entire structure of such a chiral point defect is determined by the gradient field of a harmonic function. Many of the singularities in Arnold's list have harmonic representatives; for instance, the Morse singularity of Eq. (6) is harmonic if $a=2$, and a harmonic representative for the $T_{4,4,4}$ singularity is

$\phi=\operatorname{axyz}+\frac{1}{4}\left(x^{4}+y^{4}+z^{4}-3 x^{2} y^{2}-3 y^{2} z^{2}-3 z^{2} x^{2}\right)$.

They can all be perturbed so as to be chiral following the prescription for curl eigenfields. Nonetheless, it is well 
known that not all types of critical point can occur in a harmonic function: By the maximum principle, there are no local maxima or minima. This recapitulates the content of Eliashberg and Thurston's more general result [53]; there are no chiral point defects with a radial director field.

Harmonic polynomial functions can be expressed in terms of spherical harmonics

$$
\phi=\sum_{l, m} a_{l m} r^{l} Y_{l m}
$$

For example, the harmonic Morse critical points are given by $r^{2} Y_{20}$, while the harmonic form of $T_{4,4,4}$, Eq. (28), is

$$
\sqrt{\frac{8 \pi}{105}} a \operatorname{Im} r^{3} Y_{32}+\sqrt{\frac{5 \pi}{126}} \operatorname{Re} r^{4} Y_{44}+\frac{\sqrt{\pi}}{6} r^{4} Y_{40}
$$

Similarly, a harmonic form for the charge -2 defect can be constructed in this way using $\phi=\operatorname{Im} r^{3} Y_{33}+\kappa r^{4} Y_{40}$. The chiral perturbation is also given explicitly as

$$
\mathbf{m}_{c}^{(1)}=q_{0} \sum_{l, m} \frac{a_{l m}}{l+1} r^{l} \mathbf{x} \times \nabla Y_{l m}
$$

In general, the leading spherical harmonic determines the symmetry of the defect and, in particular, the arrangement of accompanying "boundary layer defects," while higherorder terms ensure that it is isolated. In this way, defects with higher topological charges and desired symmetries can be constructed. The observed point defects of charges -1 , -2 , and -3 have symmetries such that the accompanying +1 boundary layer defects exhibit highly geometric configurations with maximal separation: antipodal points, the vertices of an equilateral triangle, and the vertices of a tetrahedron, respectively. These correspond to the energyminimizing configurations of identical point charges on the surface of a sphere interacting via the Coulomb force known as the Thomson problem [64], which provides a candidate for the symmetry of the higher-charge defects. A charge -5 defect with octahedral symmetry is captured by the function

$$
\begin{aligned}
\phi & =-\frac{\sqrt{\pi}}{6} r^{4} Y_{40}-\sqrt{\frac{5 \pi}{126}} \operatorname{Re} r^{4} Y_{44}, \\
& =\frac{3}{4}\left(x^{2} y^{2}+y^{2} z^{2}+z^{2} x^{2}\right)-\frac{1}{4}\left(x^{4}+y^{4}+z^{4}\right) .
\end{aligned}
$$

Octahedral arrangements of boundary layer defects have been observed experimentally in topological molecules consistent with unfoldings of this charge -5 defect $[20,21]$. Curiously, the bipyramidal charge -4 defect requires higher-order spherical harmonics to construct; it is captured by the function

$$
\begin{aligned}
\phi= & \frac{32}{3} \sqrt{\frac{\pi}{385}} \operatorname{Im} r^{5} Y_{53}+2 \sqrt{\frac{\pi}{17}} \tilde{\kappa} r^{8} Y_{80}, \\
= & \left(x^{2}+y^{2}-8 z^{2}\right)\left(x^{2} y-\frac{1}{3} y^{3}\right) \\
& +\tilde{\kappa}\left(z^{8}-14 z^{6}\left(x^{2}+y^{2}\right)+\frac{105}{4} z^{4}\left(x^{2}+y^{2}\right)^{2}\right. \\
& \left.-\frac{35}{4} z^{2}\left(x^{2}+y^{2}\right)^{3}+\frac{35}{128}\left(x^{2}+y^{2}\right)^{4}\right) .
\end{aligned}
$$

Its higher-order structure suggests a less stable core, which may explain why it has not yet been observed.

\section{IX. $\lambda$ LINES AND UMBILICS: DEFECTS IN THE CHOLESTERIC PITCH}

In a cholesteric, the local direction along which the director twists is called the pitch axis. It varies through the material and can have its own defects called $\lambda$ lines $[26,65]$. Loosely, the pitch axis is the direction, among all those perpendicular to the director, along which the director is rotating most rapidly. More concretely [17,66], given any direction $\mathbf{v}$ orthogonal to $\mathbf{n}$, the directional derivative $(\mathbf{v} \cdot \nabla) \mathbf{n}$ has components along $\mathbf{v}$ and along the perpendicular direction $\mathbf{n} \times \mathbf{v}$. The component along this latter direction describes a rotation of the director field along the direction $\mathbf{v}$, and the pitch axis is the direction for which this rate of rotation is maximal.

This description sits inside a broader treatment of the director gradients. At any point, there is a local symmetry group isomorphic to $S O(2)$ corresponding to rotations about the local director, and its gradients decompose into irreducible components with respect to the action of this group

$$
\begin{aligned}
\partial_{i} n_{j}= & n_{i}\left(n_{k} \partial_{k}\right) n_{j}+\frac{\nabla \cdot \mathbf{n}}{2}\left(\delta_{i j}-n_{i} n_{j}\right) \\
& +\frac{\mathbf{n} \cdot \nabla \times \mathbf{n}}{2} \epsilon_{i j k} n_{k}+\Delta_{i j} .
\end{aligned}
$$

The first term represents derivatives parallel to the director field - these are the bend distortions - while the others constitute the orthogonal gradients, $\nabla_{\perp} \mathbf{n}$. Of these, the first two are the splay and twist distortions, and the last $\Delta_{i j}$ is the deviatoric part (traceless, symmetric) of the orthogonal gradients, whose eigenvectors define principal directions of curvature for the director field. In the case where $\mathbf{n}$ is a gradient field, these directions are precisely the principal curvature directions of the surfaces that $\mathbf{n}$ is normal to.

The pitch axis in a cholesteric is conveyed by the closely related deviatoric tensor

$$
\Pi_{i j}=\Delta_{i l} \epsilon_{l j k} n_{k}
$$

obtained by composition of $\Delta_{i j}$ with the almost complex structure $\epsilon_{i j k} n_{k}$. The pitch axis can be identified with the 
positive eigenvector of $\Pi_{i j}[17,43,66]$. Defects in the pitch axis - the $\lambda$ lines of cholesterics - correspond to degeneracies of $\Pi_{i j}$ where it vanishes identically. By construction, $\Pi_{i j}$ is a rank 2 matrix, so its zeros have codimension 2 and correspond to a set of one-dimensional lines in the material known as umbilic lines [17]. The structure of $\Pi_{i j}$ around each umbilic allows it to be labeled with an index, and the full set of umbilic lines then conveys topological information about the director field; for example, it provides a means of counting the number of skyrmions or the topological charge of point defects [17]. Most commonly, this structure is represented in terms of the winding of the eigenvectors of $\Pi_{i j}$, equivalently, the pitch axis. Generically, the pitch axis has winding number $\pm 1 / 2$ around an umbilic - these are the $\lambda$ lines-but other windings are possible; the axis of a double-twist cylinder is a degenerate umbilic around which the pitch axis has winding number +1 .

Point defects in the director are associated with a confluence of umbilic lines, whose number and type are related on topological grounds to the type of point defect; for a defect of topological charge $k$, there are a total of $4|k|$ lines counted with multiplicity [17]. For chiral point defects, however, a much more detailed description can be given. As the structure of chiral point defects is determined by the gradient field of an isolated singularity, the location and types of defects in the pitch axis coincide with the structure of umbilic lines of the local level manifolds of the function describing the critical point. Passing through the critical value, the level sets change between $1+|k|$ disconnected disks and a connected surface of Euler characteristic $1-|k|$. Each disk is pierced by the axis of a double-twist cylinder $(\lambda /$ umbilic line of multiplicity 2), while further $2|k|-2$ elementary lines pierce the connected surface along directions of high symmetry. The pattern of lines for the Morse index 2, $D_{4}^{-}$, and $T_{4,4,4}$ singularities are shown in Fig. 11, with the umbilic lines indicated by white tubes obtained numerically as isosurfaces where the norm of $\Pi_{i j}$ drops below some threshold value.

Taking $D_{4}^{-}$as an example, the topological charge is -2 so that the topology requires there to be eight umbilic lines counted with multiplicity. There are three degenerate umbilic lines [double-twist cylinders; Fig. 11(d)] connecting the central defect to the +1 surface defects, which should be counted with multiplicity 2 [17] for a total of six umbilic lines. Symmetry considerations tell us that the remaining two umbilic lines [with $\lambda^{-1 / 2}$ profile; Fig. 11(e)] extend along the $z$ axis. The structure is shown in Fig. 12(a). A similar description can be given in all cases.

It is interesting to consider the behavior of umbilic lines under an unfolding of the singularity. Consider the unfoldings of $D_{4}^{-}$into a pair of charge -1 defects. Each defect produced must have four umbilic lines counted with multiplicity. With this constraint, there are two possible configurations. In one case, Fig. 12(b), one of the degenerate umbilic lines splits into a pair of $\lambda^{1 / 2}$ lines. The pair of defects produced by the unfolding each take charge of one of these $\lambda^{1 / 2}$ lines, along with one each of the two $\lambda^{-1 / 2}$ lines and two remaining degenerate umbilic lines that are connected to the original charge -2 defect, so that each defect has a total of four umbilic lines when counted with

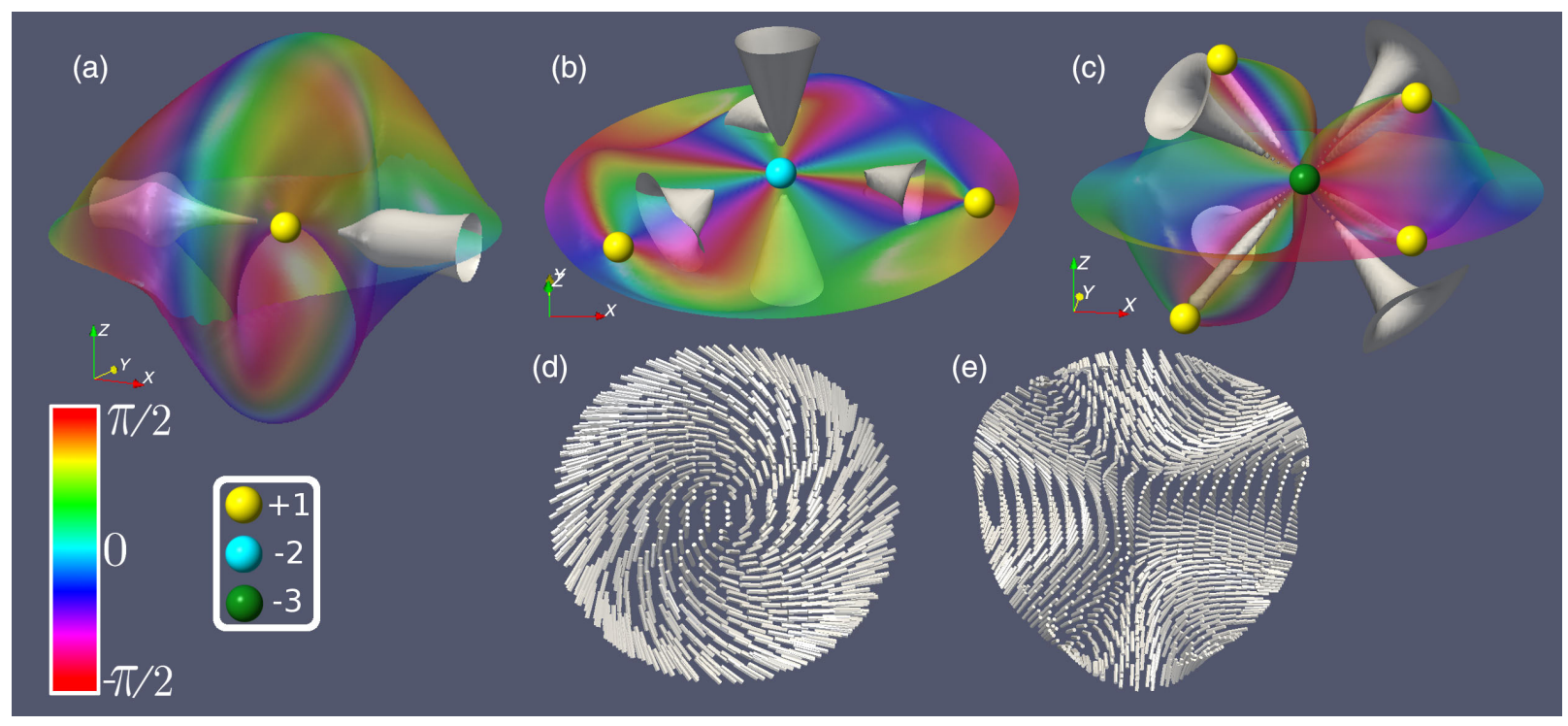

FIG. 11. Umbilic lines for the (a) Morse index 2, (b) $D_{4}^{-}$, and (c) $T_{4,4,4}$ singularities indicated by white isosurfaces. Each defect has double-twist cylinders connecting it to the charge +1 surface defects. The remaining umbilics are $\lambda^{-1 / 2}$ lines that terminate on the droplet surface. Typical cross-sectional profiles for the umbilics are shown in (d) double-twist cylinders and (e) $\lambda^{-1 / 2}$ lines. In all panels, defects are highlighted and color coded according to topological charge. 


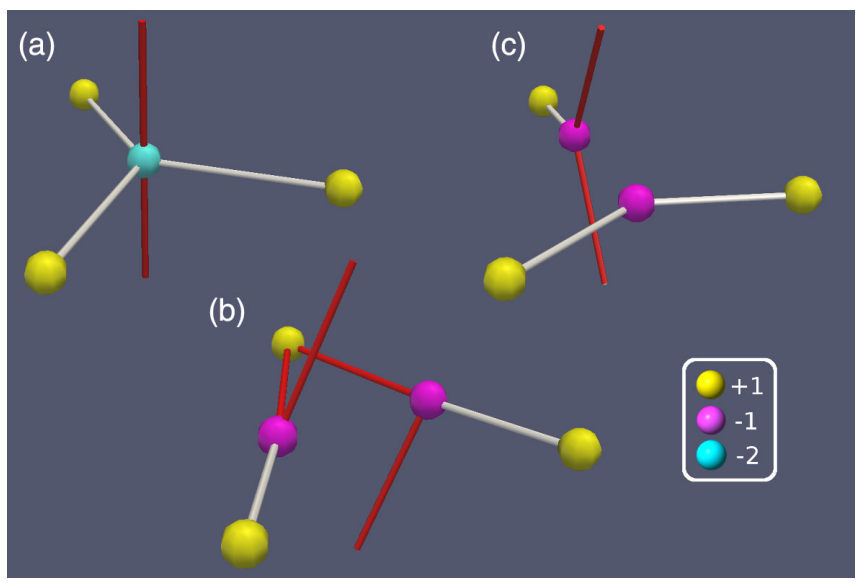

FIG. 12. (a) Position of the umbilic lines for the $D_{4}^{-}$singularity. (b),(c) Possible configurations of umbilic lines after an unfolding into a pair of charge -1 defects. In each case, the white tubes indicate degenerate umbilic lines with multiplicity 2 (the axes of double-twist cylinders), while the red tubes indicate generic umbilic lines $\left(\lambda^{-1 / 2}\right.$ lines).

multiplicity. The other case shown in Fig. 12(c) has one defect connected to a pair of degenerate umbilic lines, while the other defect has a single degenerate umbilic line and both of the $\lambda^{-1 / 2}$ lines.

Both cases can be realized in numerical simulations by initializing the simulation with the model given by Eq. (14) with unfolding parameters $c_{1}, c_{3}=0$, and $c_{2} \neq 0$. When $c_{2}>0$, we obtain a $\mathrm{V}$-shaped unfolding, and the configuration of umbilic lines is as in Fig. 12(b). When $c_{2}<0$, we obtain a T-shaped unfolding, and the configuration of umbilic lines produced is Fig. 12(c). A similar description can be given for the unfoldings of $T_{4,4,4}$.

\section{DISCUSSION}

It is surprising, to us at least, that a systematic description of the structure and properties of point defects in cholesterics, or other chiral vector fields, has not been given previously. The structure is nontrivial and different from that of nematic hedgehogs because generic point defects are incompatible with a uniform sense of twist around them. In order to create uniform handedness, chiral point defects have to adopt the structure of the gradient field of an isolated singularity, with the nonzero twist coming from subleading terms. This means that point defects in cholesterics, as well as in chiral ferromagnets and Beltrami fields (force-free magnetic fields, or helical flows), can be labeled by the type of singularity, in addition to the coarser topological degree, or hedgehog charge.

This link with singularity theory suggests that the richness of that subject can be harnessed to direct future developments. For instance, the structure of topological molecules observed in experiments is strikingly captured by the unfoldings of complex singularities, which provides a ready-made framework for future systematic analysis. At the same time, not all aspects of singularity theory have an immediate realization in cholesterics, leaving open questions about the flavor of singularity theory that is involved here. For instance, it is not clear whether the full list of singularities all occur or in an order predicted by their codimension. As examples, the lowest codimension singularity with topological charge -3 is $X_{9}$ (codimension 7) and not $T_{4,4,4}$, which is observed in experiments. Likewise, the simple singularity $E_{7}$ has charge -1 and is found to be stable in numerical simulations with a structure that differs from the Morse index 1 defect, although so far it has not been identified in any experiments.

The mathematical result of Eliashberg and Thurston represents a fundamental physical property of chiral materials that has not previously been recognized. We bring its application to physical phenomena for the first time here and show that it serves also to constrain the structure of chiral point defects. In a loosest sense, it implies that the addition of a small amount of chiral dopant to a radial nematic hedgehog needs to be considered in nonperturbative terms. More generally, it reveals that geometric constraints on the structure of the director field can be used to topologically frustrate the twist of chiral materials and create localized topological solitons of reversed handedness. We describe the simplest examples, but there is clearly great flexibility in controlling the geometry of domains and interfaces, or the arrangement of point defects, so as to control these novel chiral solitons and create interesting new textures.

A natural extension of the present work is to initiate a theory of the structure and properties of disclination loops in cholesterics. It may be anticipated that they too will require specific local forms in order to be surrounded by a single sense of twist and may encounter analogous topological obstructions to achieving a uniform handedness. For their counterparts in nematics, the connections between point defects and disclination loops proved essential for understanding the latter [30]; the same can be expected for cholesterics.

We should stress that while we focus on a presentation of point defects in cholesteric liquid crystals, our analysis applies more widely to all types of chiral ordered media. Thus, the same phenomena will also be observed in the defects of chiral ferromagnets. The simplest examples of these have been reported recently [40], and indeed the structure found matches exactly that of the chiral Morse defects we describe here. Beltrami fields have found applications in force-free magnetic fields and in certain idealized inviscid fluid flows [59,60]. There, too, the general results we describe for chiral point defects apply to the structure of isolated zeros.

Finally, the properties of chiral point defects afford a fresh perspective on the storied contrast between smectics and cholesterics; sometimes this emphasizes similarities, 
for instance, they have the same elasticity [67], but usually the focus is on the sense in which they are antithetical. The nonvanishing of the twist is precisely the conditionFrobenius integrability theorem-that the director field is not the normal to any set of layers and provides a geometric characterization of cholesterics [68]. Conversely, the expulsion of twist from a smectic is a hallmark of that phase, famously analogous to the Meissner effect [18]. Intriguingly, in the presence of defects, the division between the two is diminished as the local structure of chiral point defects is required to converge towards that of a smectic.

\section{CONCLUSION}

Chiral materials display a rich assortment of phenomena arising from the competition between an energetic preference for twist and confinement or surface boundary conditions. The resulting textures, in cholesteric liquid crystals and helimagnets, are often both geometric and topological, including skyrmion phases, knot solitons, and configurations of topological defects, and offer prospects in soft photonics, metamaterials, and spintronics.

The experiments we focus on here concern point defects in spherical droplets of cholesteric liquid crystal. Defects are observed both close to the droplet surface and deep in its interior; we show that generic defects are incompatible with a uniform sense of chiral twisting for topological reasons, furnishing an explanation for the distinction between the boundary layer and interior defects. The chiral defects in the droplet interior include examples with topological charges -2 and -3 , not seen in other systems, and arrangements of multiple defects reminiscent of chemical molecules. The theory we develop identifies these structures with the gradient field of an isolated critical point in a function. This identification gives a more refined description than by the topological charge alone and accounts for the structure and symmetry of the defects with high topological charge, as well as the arrangements of topological molecules.

Our results identify the mathematical methods relevant to the description of defects in chiral materials, as well as their surface boundary behavior. The understanding and control of defects proves central to numerous advances in condensed matter physics, and our work can contribute to a fuller picture of chiral media and their properties by bringing powerful techniques from modern geometry and topology to the forefront.

\section{ACKNOWLEDGMENTS}

G.P. A. acknowledges beneficial discussions with Michael Berry and David Chillingworth on singularity theory and Randy Kamien on defects in the pitch axis. We are grateful to a referee for bringing Ref. [50] to our attention. This work is supported by the UK EPSRC through Grant No. EP/L015374/1 (J.P. and G. P. A.) and the Slovenian Research Agency (ARRS) under Contracts
No. P1-0099 (G. P., S. Č., and I. M.) and No. J1-9149 (S. C..). This work results within the collaboration of the COST Action CA17139.

[1] G. I. Taylor, The Mechanism of Plastic Deformation of Crystals. Part I. Theoretical, Proc. R. Soc. A 145, 362 (1934).

[2] P. Poulin, H. Stark, T. C. Lubensky, and D. A. Weitz, Novel Colloidal Interactions in Anisotropic Fluids, Science 275, 1770 (1997).

[3] M. Škarabot, M. Ravnik, S. Žumer, U. Tkalec, I. Poberaj, D. Babič, N. Osterman, and I. Muševič, Two-Dimensional Dipolar Nematic Colloidal Crystals, Phys. Rev. E 76, 051406 (2007).

[4] I. Muševič, M. Škarabot, U. Tkalec, M. Ravnik, and S. Žumer, Two-Dimensional Nematic Colloidal Crystals SelfAssembled by Topological Defects, Science 313, 954 (2006).

[5] I. Muševič, Liquid Crystal Colloids (Springer International Publishing, Cham, 2017), pp. 119-148.

[6] X. Wang, D. S. Miller, E. Bukusoglu, J. J. de Pablo, and N.L. Abbott, Topological Defects in Liquid Crystals as Templates for Molecular Self-Assembly, Nat. Mater. 15, 106 (2016).

[7] A. A. Abrikosov, On the Magnetic Properties of Superconductors of the Second Group, Zh. Eksp. Teor. Fiz. 32, 1442 (1957) [Sov. Phys. JETP 5, 1174 (1957)].

[8] V.L. Berezinskiĭ, Destruction of Long-Range Order in One-Dimensional and Two-Dimensional Systems Having a Continuous Symmetry Group I. Classical Systems, Zh. Eksp. Teor. Fiz. 59, 907 (1970) [Sov. Phys. JETP 32, 493 (1971)].

[9] J. M. Kosterlitz and D. J. Thouless, Ordering, Metastability and Phase Transition in Two-Dimensional Systems, J. Phys. C 6, 1181 (1973).

[10] S. R. Renn and T.C. Lubensky, Abrikosov Dislocation Lattice in a Model of the Cholesteric-to-Smectic-A Transition, Phys. Rev. A 38, 2132 (1988).

[11] H. Kikuchi, Liquid Crystalline Blue Phases, in Liquid Crystalline Functional Assemblies and Their Supramolecular Structures. Structure and Bonding, edited by T. Kato (Springer, Berlin, 2007), pp. 99-117.

[12] I. Chuang, R. Durrer, N. Turok, and B. Yurke, Cosmology in the Laboratory: Defect Dynamics in Liquid Crystals, Science 251, 1336 (1991).

[13] T. B. Saw, A. Doostmohammadi, V. Nier, L. Kocgozlu, S. Thampi, Y. Toyama, P. Marcq, C. T. Lim, J. M. Yeomans, and B. Ladoux, Topological Defects in Epithelia Govern Cell Death and Extrusion, Nature (London) 544, 212 (2017).

[14] Y. Bouligand, Liquid Crystals and Biological Morphogenesis: Ancient and New Questions, C. R. Chim. 11, 281 (2008).

[15] A. Nych, J.-I. Fukuda, U. Ognysta, S. Žumer, and I. Muševič, Spontaneous Formation and Dynamics of HalfSkyrmions in a Chiral Liquid-Crystal Film, Nat. Phys. 13, 1215 (2017). 
[16] P. J. Ackerman and I. I. Smalyukh, Diversity of Knot Solitons in Liquid Crystals Manifested by Linking of Preimages in Torons and Hopfions, Phys. Rev. X 7, 011006 (2017).

[17] T. Machon and G. P. Alexander, Umbilic Lines in Orientational Order, Phys. Rev. X 6, 011033 (2016).

[18] P. G. de Gennes, An Analogy between Superconductors and Smectics A, Solid State Commun. 10, 753 (1972).

[19] G. Posnjak, S. Čopar, and I. Muševič, Points, Skyrmions and Torons in Chiral Nematic Droplets, Sci. Rep. 6, 26361 (2016).

[20] G. Posnjak, S. Čopar, and I. Muševič, Hidden Topological Constellations and Polyvalent Charges in Chiral Nematic Droplets, Nat. Commun. 8, 14594 (2017).

[21] G. Posnjak, in Topological Formations in Chiral Nematic Droplets (Springer, Cham, 2018).

[22] F. R. N. Nabarro, Singular Lines and Singular Points of Ferromagnetic Spin Systems and of Nematic Liquid Crystals, J. Phys. France 33, 1089 (1972).

[23] M. V. Kurik and O. D. Lavrentovich, Negative-Positive Monopole Transitions in Cholesteric Liquid Crystals, JETP Lett. 35, 444 (1982).

[24] G. E. Volovik and O. D. Lavrentovich, Topological Dynamics of Defects: Boojums in Nematic Drops, Zh. Eksp. Teor. Fiz. 85, 1997 (1983) [Sov. Phys. JETP 58, 1159 (1983)].

[25] M. Kléman and O. D. Lavrentovich, Topological Point Defects in Nematic Liquid Crystals, Philos. Mag. 86, 4117 (2006).

[26] O. D. Lavrentovich and M. Kléman, Cholesteric Liquid Crystals: Defects and Topology, in Chirality in Liquid Crystals. Partially Ordered Systems, edited by H.S. Kitzerow and C. Bahr (Springer, New York, 2001).

[27] A. Pargellis, N. Turok, and B. Yurke, MonopoleAntimonopole Annihilation in a Nematic Liquid Crystal, Phys. Rev. Lett. 67, 1570 (1991).

[28] I.-H. Lin, D. S. Miller, P. J. Bertics, C. J. Murphy, J. J. de Pablo, and N.L. Abbott, Endotoxin-Induced Structural Transformations in Liquid Crystalline Droplets, Science 332, 1297 (2011).

[29] H.-G. Lee, S. Munir, and S.-Y. Park, Cholesteric Liquid Crystal Droplets for Biosensors, ACS Appl. Mater. Interfaces 8, 26407 (2016).

[30] G. P. Alexander, B. G. Chen, E. A. Matsumoto, and R. D. Kamien, Colloquium: Disclination Loops, Point Defects, and All That in Nematic Liquid Crystals, Rev. Mod. Phys. 84, 497 (2012).

[31] B. Senyuk, Q. Liu, S. He, R. D. Kamien, R. B. Kusner, T. C. Lubensky, and I. I. Smalyukh, Topological Colloids, Nature (London) 493, 200 (2013).

[32] E. Pairam, J. Vallamkondu, V. Koning, B. C. van Zuiden, P. W. Ellis, M. A. Bates, V. Vitelli, and A. Fernandez-Nieves, Stable Nematic Droplets with Handles, Proc. Natl. Acad. Sci. U.S.A. 110, 9295 (2013).

[33] S. Čopar and S. Žumer, Nematic Braids: Topological Invariants and Rewiring of Disclinations, Phys. Rev. Lett. 106, 177801 (2011).

[34] S. Čopar and S. Žumer, Nematic Disclinations as Twisted Ribbons, Phys. Rev. E 84, 051702 (2011).

[35] R. Thom, Structural Stability and Morphogenesis (Westview Press, Boulder, 1994).
[36] M. V. Berry, Waves and Thom's Theorem, Adv. Phys. 25, 1 (1976).

[37] T. Poston and I. Stewart, Catastrophe Theory and Its Applications (Dover Publications, Inc., New York, 1978).

[38] J.-I. Fukuda and S. Žumer, Quasi-Two-Dimensional Skyrmion Lattices in a Chiral Nematic Liquid Crystal, Nat. Commun. 2, 246 (2011).

[39] A. O. Leonov, I. E. Dragunov, U. K. Rößler, and A. N. Bogdanov, Theory of Skyrmion States in Liquid Crystals, Phys. Rev. E 90, 042502 (2014).

[40] P. Milde, D. Kohler, J. Seidel, L. M. Eng, A. Bauer, A. Chacon, J. Kindervater, S. Mühlbauer, C. Pfleiderer, S. Buhrandt, C. Schutte, and A. Rosch, Unwinding of a Skyrmion Lattice by Magnetic Monopoles, Science 340, 1076 (2013).

[41] B. G. Chen, P. J. Ackerman, G. P. Alexander, R. D. Kamien, and I. I. Smalyukh, Generating the Hopf Fibration Experimentally in Nematic Liquid Crystals, Phys. Rev. Lett. 110, 237801 (2013).

[42] B. G. Chen, Topological Defects in Nematics and Smectic Liquid Crystals, Ph.D. dissertation, University of Pennsylvania, 2012.

[43] G. P. Alexander, Topology in Liquid Crystal Phases, in The Role of Topology in Materials, edited by S. Gupta and A. Saxena (Springer, Cham, 2018).

[44] J. Milnor, Morse Theory (Princeton University Press, Princeton, NJ, 1963).

[45] V. I. Arnold, Index of a Singular Point of a Vector Field, the Petrovskii-Oleinik Inequality, and Mixed Hodge Structures, Funkts. Anal. Prilozh. 12, 1 (1978) [Funct. Anal. Appl. 12, 1 (1978)].

[46] S. Čopar and S. Žumer, Topological and Geometric Decomposition of Nematic Textures, Phys. Rev. E 85, 031701 (2012).

[47] F. C. Frank, I. Liquid Crystals. On the Theory of Liquid Crystals, Discuss. Faraday Soc. 25, 19 (1958).

[48] M. Ravnik and S. Žumer, Landau-de Gennes Modelling of Nematic Liquid Crystal Colloids, Liq. Cryst. 36, 1201 (2009).

[49] I. I. Smalyukh, S. V. Shiyanovskii, and O. D. Lavrentovich, Three-Dimensional Imaging of Orientational Order by Fluorescence Confocal Polarizing Microscopy, Chem. Phys. Lett. 336, 88 (2001).

[50] O. D. Lavrentovich and E. M. Terent'ev, Phase Transition Altering the Symmetry of Topological Point Defects (Hedgehogs) in a Nematic Liquid Crystal, Zh. Eksp. Teor. Fiz. 91, 2084 (1986) [Sov. Phys. JETP 64, 1237 (1986)].

[51] A. Rüdinger and H. Stark, Twist Transition in Nematic Droplets: A Stability Analysis, Liq. Cryst. 26, 753 (1999).

[52] R. James and J.-I. Fukuda, Twist Transition of Nematic Hyperbolic Hedgehogs, Phys. Rev. E 89, 042501 (2014).

[53] Y. M. Eliashberg and W.P. Thurston, Confoliations (American Mathematical Society, Providence, 1998).

[54] P. J. Ackerman and I. I. Smalyukh, Reversal of Helicoidal Twist Handedness near Point Defects of Confined Chiral Liquid Crystals, Phys. Rev. E 93, 052702 (2016).

[55] J. Martinet, Sur les singularités des formes différentielles, Ann. Inst. Fourier 20, 95 (1970).

[56] M. Zhitomirskiı̌, Typical Singularities of Differential 1-Forms and Pfaffian Equations (American Mathematical Society, Providence, 1992). 
[57] V. I. Arnold, V. V. Goryunov, O. V. Lyashko, and V. A. Vasil'ev, Singularity Theory I (Springer-Verlag, Berlin, 1998).

[58] V. I. Arnold, Normal Forms for Functions near Degenerate Critical Points, the Weyl Groups of $A_{k}, D_{k}, E_{k}$ and Lagrangian Singularities, Funkts. Anal. Prilozh. 6, 3 (1972) [Funct. Anal. Appl. 6, 254 (1972)].

[59] S. Chandrasekhar and P. C. Kendall, On Force-Free Magnetic Fields, Astrophys. J. 126, 457 (1957).

[60] J. Etnyre and R. Ghrist, Contact Topology and Hydrodynamics: I. Beltrami Fields and the Seifert Conjecture, Nonlinearity 13, 441 (2000).

[61] D. C. Wright and N. D. Mermin, Crystalline Liquids: The Blue Phases, Rev. Mod. Phys. 61, 385 (1989).

[62] S. Tewari, D. Belitz, and T. R. Kirkpatrick, Blue Quantum Fog: Chiral Condensation in Quantum Helimagnets, Phys. Rev. Lett. 96, 047207 (2006).

[63] B. Binz, A. Vishwanath, and V. Aji, Theory of the Helical Spin Crystal: A Candidate for the Partially Ordered State of MnSi, Phys. Rev. Lett. 96, 207202 (2006).
[64] J. J. Thomson, XXIV. On the Structure of the Atom: An Investigation of the Stability and Periods of Oscillation of a Number of Corpuscles Arranged at Equal Intervals around the Circumference of a Circle; with Application of the Results to the Theory of Atomic Structure, Philos. Mag. Ser. 6 7, 237 (1904).

[65] D. A. Beller, T. Machon, S. Čopar, D. M. Sussman, G. P. Alexander, R. D. Kamien, and R. A. Mosna, Geometry of the Cholesteric Phase, Phys. Rev. X 4, 031050 (2014).

[66] T. J. Machon, Aspects of Geometry and Topology in Liquid Crystalline Phases, Ph.D. dissertation, University of Warwick, 2016.

[67] J. Toner and D. R. Nelson, Smectic, Cholesteric and Rayleigh-Benard Order in Two Dimensions, Phys. Rev. B 23, 316 (1981).

[68] T. Machon, Contact Topology and the Structure and Dynamics of Cholesterics, New J. Phys. 19, 113030 (2017). 